\title{
Searching Cycle-Disjoint Graphs
}

Boting Yang, Runtao Zhang, and Yi Cao Technical Report TR-CS 2006-5

March, 2006

(C) Boting Yang, Runtao Zhang, and Yi Cao

Department of Computer Science

University of Regina

Regina, Saskatchewan, CANADA

S4S 0A2

ISBN 0-7731-0571-9 (print)

ISBN 0-7731-0572-7 (on-line) 


\title{
Searching Cycle-Disjoint Graphs*
}

\author{
Boting Yang, Runtao Zhang, Yi Cao \\ Department of Computer Science \\ University of Regina
}

February 26, 2006

\begin{abstract}
In this paper, we consider the edge searching problem on cycle-disjoint graphs. We first improve the running time of the algorithm to compute the vertex separation and the optimal layout of a unicyclic graph, which is given by Ellis et al. (2004), from $O(n \log n)$ to $O(n)$. By a linear-time transformation, we can compute the edge search number of a unicyclic graph in linear time. We also propose an $O(n)$ time algorithm to compute the edge search number and the optimal edge search strategy of a cycle-disjoint graph in which every cycle has at most three vertices with degree more than two. We show how to compute the search number for a $k$-ary cycle-disjoint graph. We also present some results on approximation algorithms.

Keywords: edge searching, vertex separation, cycle-disjoint graph, unicyclic graph.
\end{abstract}

\section{Introduction}

The edge searching problem is to find the minimum number of searchers to capture an intruder that is hiding on vertices or edges of a graph [10]. There are other searching models besides edge searching, but in this paper we mainly consider the edge searching problem. For this reason, we will use "search" instead of "edge search" for simplicity.

Let $G$ be a graph without loops and multiple edges. Initially, all vertices and edges of $G$ are contaminated, which means an intruder can hide on any vertices or anywhere along edges. There are three actions for searchers: (1) place a searcher on a vertex; (2) remove a searcher from a vertex; (3) slide a searcher along an edge from one end vertex to the other. A search strategy is a sequence of actions designed so that the final action leaves all edges of $G$ cleared. An edge $u v$ in $G$ can be cleared in one of the following two ways by a sliding action: (1) two searchers are located on vertex $u$, and one of them slides along $u v$ from $u$ to $v$; or (2) a searcher is located on vertex $u$, where all edges incident with $u$, other than $u v$, are already cleared, and the searcher slides from $u$ to $v$. The intruder can slide along a path that contains no searcher at a great speed at any time. The minimum number of searchers required to clear $\mathrm{G}$ is called the search number of $G$, denoted by $s(G)$. A search strategy for $G$ is optimal if this strategy clears $G$ using $s(G)$ searchers.

Let $S$ be a search strategy for a graph $G$ and let $E(i)$ be the set of cleared edges just after action $i . S$ is said to be monotonic if $E(i) \subseteq E(i+1)$ for each $i$. LaPaugh [11] proved that for any connected graph $G$, allowing recontamination cannot reduce the search number. Thus, throughout this paper, we only need to consider monotonic search strategies. For this reason, we will use "search strategy" instead of "monotonic search strategy" for simplicity.

Megiddo et al. [12] showed that determining the search number of a graph $G$ is NP-complete. They also gave an $O(n)$ time algorithm to compute the search number of a tree and an $O(n \log n)$ time algorithm to find the optimal search strategy, where $n$ is the number of vertices in the tree. Peng et al. [14] proposed an $O(n)$ time algorithm to compute the optimal search strategy of a tree.

Search numbers are closely related to several other important graph parameters. Ellis et al. [6] proved that for any connected undirected graph $G$ and its 2-expansion $G^{\prime}$, the vertex operation of $G^{\prime}$ equals the search number of $G^{\prime}$, which has the same search number as $G$. Kinnersley [9] showed that vertex separation is identical to pathwidth, an important measure of graph structure.

A layout of a connected graph $G(V, E)$ is a one to one mapping $L: V \rightarrow\{1,2, \ldots,|V|\}$. Let $V_{L}(i)=\{x$ : $x \in V(G)$, and there exists $y \in V(G)$ such that the edge $x y \in E(G), L(x) \leq i$ and $L(y)>i\}$. The vertex separation of $G$ with respect to $L$, denoted by $\operatorname{vs}_{L}(G)$, is defined $\operatorname{as~} \operatorname{vs}_{L}(G)=\max \left\{\left|V_{L}(i)\right|: 1 \leq i \leq|V(G)|\right\}$. The vertex separation of $G$ is defined as $\operatorname{vs}(G)=\min \left\{\operatorname{vs}_{L}(G): L\right.$ is a layout of $\left.G\right\}$. We say that $L$ is an optimal layout if $\operatorname{vs}_{L}(G)=\mathrm{vs}(G)$. Ellis et al. [6] proved that $\operatorname{vs}(G) \leq s(G) \leq \mathrm{vs}(G)+2$ for any connected

${ }^{*}$ This research was supported in part by NSERC and MITACS. 
undirected graph $G$. They also proposed an algorithm to compute the vertex separation of a tree in $O(n)$ time. Based on this algorithm, Ellis and Markov [7] gave an $O(n \log n)$ time algorithm to compute the vertex separation and the corresponding optimal layout of a unicyclic graph.

Bodlaender and Kloks [3] gave a polynomial time algorithm for computing the pathwidth of a graph with constant treewidth. Since the search number of a graph equals the pathwidth of its 2-expansion, we know that the search number of a graph with constant treewidth is polynomial time computable. However, the exponent in the running time of this algorithm is very large. Even for a graph with treewidth two, it takes $\Omega\left(n^{11}\right)$ time. Bodlaender and Fomin [4] introduced an $O(n)$ time approximation algorithm to compute the pathwidth of an outerplanar graph, a class of graphs with treewidth two. The approximation ratio of their algorithm is 2. Finding efficient algorithms for computing the search number of a graph with constant treewidth continues to be a challenge.

All graphs in this paper are finite without loops and multiple edges. A graph $G$ is called a cycle-disjoint graph $(C D G)$ if it is connected and no pair of cycles in $G$ share a vertex. If every cycle of a CDG $G$ has at most three vertices with degree more than two, then we call $G$ a 3-cycle-disjoint graph (3CDG). If a vertex or an edge is on a cycle of $G$, it is called, respectively, a cycle vertex or a cycle edge.

Our motivation is to find an efficient algorithm for computing the search number of a graph with treewidth at most two. We have successfully found an $O(n)$ time algorithm for a unicyclic graph. Then we tried to extend this algorithm to CDGs. However, we found the necessary structural information of CDGs is much more complicated than that of unicyclic graphs. Finally, we managed to develop an $O(n)$ time algorithm for 3CDGs. We also found the search numbers of $k$-ary CDGs that is a class of CDGs with well balanced structures.

This paper is organized as follows. In Section 2, we improve Ellis and Markov's algorithm in [7] from $O(n \log n)$ to $O(n)$. In Section 3, we propose a linear time algorithm to compute the search number and the optimal search strategy of a 3-cycle-disjoint graph using the labeling method. In Section 4, we show how to compute the search number of a $k$-ary cycle-disjoint graph. In Section 5 , we investigate approximation algorithms, and finally in Section 6, we discuss issues arising from these results.

\section{Unicyclic graphs}

Ellis and Markov [7] proposed an $O(n \log n)$ algorithm to compute the vertex separation and the optimal layout of a unicyclic graph using the labeling method. In this section we will give an improved algorithm that can do the same work in $O(n)$ time. All definitions and notation in this section are from [7]. Their algorithm consists of three functions: main, vs_uni and vs_reduced_uni (see Fig. 28, 29 and 30 in [7] for their descriptions).

Let $U$ be a unicyclic graph and $e$ be a cycle edge of $U$. The function main, first computes the vertex separation of the tree $U-e$, and then invokes function vs_uni to decide whether $\operatorname{vs}(U)=\operatorname{vs}(U-e)$. vs_uni is a recursive function that has $O(\log n)$ depth, and in each iteration it computes the vertex separation of a reduced tree $U^{\prime}-e$ and this takes $O(n)$ time. Thus, the running time of vs_uni is $O(n \log n)$. vs_uni may invoke the function vs_reduced_uni to decide whether a unicyclic graph $U$ is $k$-conforming. vs_reduced_uni is also a recursive function that has $O(\log n)$ depth, and in each iteration it may compute the vertex separation of $T_{1}[a]$ and $T_{1}[b]$ and this takes $O(n)$ time. Hence, the running time of vs_reduced_uni is also $O(n \log n)$.

We will modify all three functions. The main improvement of our algorithm is to preprocess the input of both vs_uni and vs_reduced_uni such that we can achieve $O(n)$ time. Refer to [7] for the linear time algorithm to compute the vertex separation and the optimal layout of a tree. The following is our improved algorithm, which computes the vertex separation and the optimal layout of a unicyclic graph $U$.

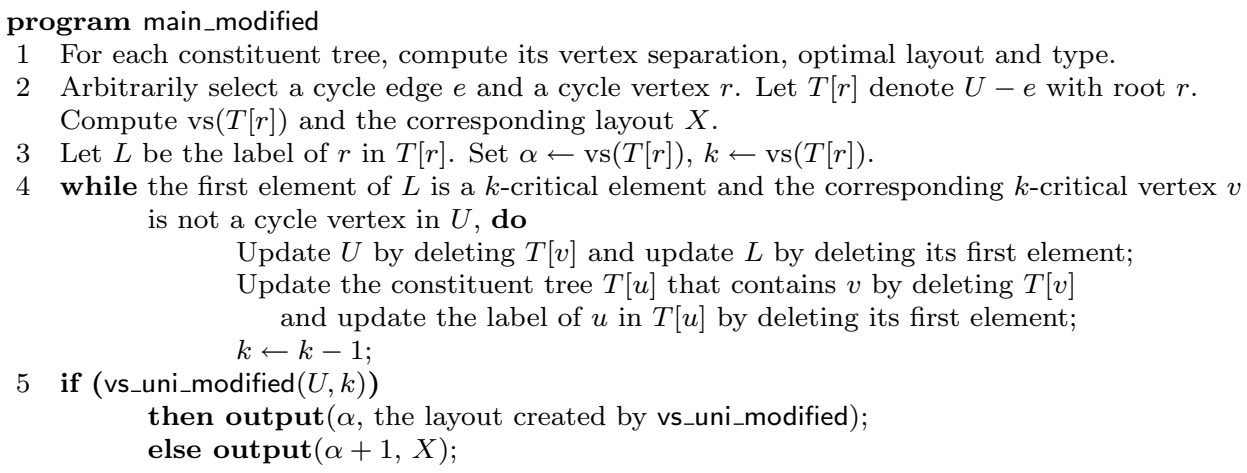


function vs_uni_modified $(U, k)$ : Boolean

Case 1: $U$ has one $k$-critical constituent tree;

compute vs $\left(T^{\prime}\right)$;

if $\operatorname{vs}\left(T^{\prime}\right)=k$, then return (false) else return (true);

Case 2: $U$ has three or more non-critical $k$-trees;

return (false);

Case 3: $U$ has exactly two non-critical $k$-trees $T_{i}$ and $T_{j}$; compute $\operatorname{vs}\left(T_{1}[a]\right), \operatorname{vs}\left(T_{1}[b]\right), \operatorname{vs}\left(T_{2}[c]\right)$ and $\operatorname{vs}\left(T_{2}[d]\right)$;

/* Assume that vs $\left(T_{1}\right) \geq \operatorname{vs}\left(T_{2}\right)$.*/

/* Let $L_{a}$ be the label of $a$ in $T_{1}[a]$, and $L_{b}$ be the label of $b$ in $T_{1}[b]$. */

/* Let $L_{c}$ be the label of $c$ in $T_{2}[c]$, and $L_{d}$ be the label of $d$ in $T_{2}[d]$. */

${ }^{*}$ Let $U^{\prime}$ be $U$ minus the bodies of $T_{i}$ and $T_{j} .{ }^{*} /$

return (vs_reduced_uni_modified $\left(U^{\prime}, L_{a}, L_{b}, L_{c}, L_{d}, k\right)$ )

Case 4: $U$ has exactly one non-critical $k$-tree $T_{i}$;

$/^{*}$ let $q$ be the number of $(k-1)$-trees that is not type NC. */

Case 4.1: $\quad 0 \leq q \leq 1$

return (true);

Case 4.2: $q=2$;

for each tree $T_{j}$ from among the two $(k-1)$-trees, do

compute the corresponding $\operatorname{vs}\left(T_{1}[a]\right), \operatorname{vs}\left(T_{1}[b]\right), \operatorname{vs}\left(T_{2}[c]\right)$ and $\operatorname{vs}\left(T_{2}[d]\right)$

if (vs_reduced_uni_modified $\left(U^{\prime}, L_{a}, L_{b}, L_{c}, L_{d}, k\right)$ ) then return (true);

* $U^{\prime}$ is equal to $U$ minus the bodies of $T_{i}$ and $T_{j}$.

Case 4.3: $q=3$;

turn (false);

for each tree $T_{j}$ from among the three $(k-1)$-trees, do

compute the corresponding $\operatorname{vs}\left(T_{1}[a]\right), \operatorname{vs}\left(T_{1}[b]\right), \operatorname{vs}\left(T_{2}[c]\right)$ and $\operatorname{vs}\left(T_{2}[d]\right)$;

if (vs_reduced_uni_modified $\left(U^{\prime}, L_{a}, L_{b}, L_{c}, L_{d}, k\right)$ ) then return (true);

$/^{*} U^{\prime}$ is equal to $U$ minus the bodies of $T_{i}$ and $T_{j} . * /$

Case 4.4: $\quad q \geq 4$;

return (false);

return (false);

Case 5: $U$ has no $k$-trees;

$/^{*}$ let $q$ be the number of $(k-1)$-trees that is not type NC. */

Case 5.1: $0 \leq q \leq 2$

return (true);

Case 5.2: $q=3$;

for each choice of two trees $T_{i}$ and $T_{j}$ from among the three $(k-1)$-trees, do compute the corresponding $\operatorname{vs}\left(T_{1}[a]\right), \operatorname{vs}\left(T_{1}[b]\right), \operatorname{vs}\left(T_{2}[c]\right)$ and $\operatorname{vs}\left(T_{2}[d]\right)$; if (vs_reduced_uni_modified $\left(U^{\prime}, L_{a}, L_{b}, L_{c}, L_{d}, k\right)$ ) then return (true); return (false);

/ $U^{\prime}$ is equal to $U$ minus the bodies of $T_{i}$ and $T_{j}$. */

Case 5.3: $q=4$;

for each choice of two trees $T_{i}$ and $T_{j}$ from among the four $(k-1)$-trees, do compute the corresponding vs $\left(T_{1}[a]\right), \operatorname{vs}\left(T_{1}[b]\right), \operatorname{vs}\left(T_{2}[c]\right)$ and $\operatorname{vs}\left(T_{2}[d]\right)$ if (vs_reduced_uni_modified $\left(U^{\prime}, L_{a}, L_{b}, L_{c}, L_{d}, k\right)$ ) then return (true);

Case 5.4: $q \geq 5$ /* $U^{\prime}$ is equal to $U$ minus the bodies of $T_{i}$ and $T_{j}$.*/

return (false);

return (false).

function vs_reduced_uni_modified $\left.\left(U, L_{a}, L_{b}, L_{c}, L_{d}, k\right)\right)$ : Boolean

$/^{*}$ Let $a_{1}, b_{1}, c_{1}, d_{1}$ be the first elements of $L_{a}, L_{b}, L_{c}, L_{d}$ respectively. */

$/^{*}$ Let $\left|a_{1}\right|,\left|b_{1}\right|,\left|c_{1}\right|,\left|d_{1}\right|$ be the value of $a_{1}, b_{1}, c_{1}, d_{1}$ respectively. We assume that $\left|a_{1}\right| \geq\left|c_{1}\right|$. */

Case 1: $\left|a_{1}\right|=k$;

return (false).

Case $2:\left|a_{1}\right|<k-1$

return (true)

Case 3: $\left|a_{1}\right|=k-1$;

if both $a_{1}$ and $b_{1}$ are $(k-1)$-critical elements,

then

${ }^{*}$ Let $u$ be the $(k-1)$-critical vertex in $T_{1}[a]$

and let $v$ be the $(k-1)$-critical vertex in $T_{1}[b] . * /$

if $u=v$ and $u$ is not a cycle vertex,

then

update $L_{a}$ and $L_{b}$ by deleting their first elements;

update $U$ by deleting $T[u]$;

update the label of the root of the constituent tree containing $u$ by deleting its first element;

if $\left|c_{1}\right|$ is greater than the value of the first element in current $L_{a}$,

then return (vs_reduced_uni_modified $\left(U, L_{c}, L_{d}, L_{a}, L_{b}, k-1\right.$ ).

else return (vs_reduced_uni_modified $\left(U, L_{a}, L_{b}, L_{c}, L_{d}, k-1\right)$.

else $/^{*}(u=v$ and $u$ is a cycle vertex $)$ or $(u \neq v) * /$

return $\left(T_{2}\right.$ contains no $k-1$ types other than NC constituents);

else return ((neither $a_{1}$ nor $d_{1}$ is $(k-1)$-critical element)

or (neither $b_{1}$ nor $c_{1}$ is $(k-1)$-critical element)). 
Lemma 2.1 Let $U$ be a unicyclic graph, e be a cycle edge and $r$ be a cycle vertex in $U$. Let $T[r]$ denote the rooted tree $U-e$ with root $r$. If $\operatorname{vs}(T[r])=k$, then $U$ has a $k$-constituent tree of type $C b$ if and only if the first element in the label of $r$ in $T[r]$ is a $k$-critical element and the corresponding $k$-critical vertex is not a cycle vertex.

Proof. $(\Rightarrow)$. Let $T^{\prime}$ be the $k$-constituent tree of type $\mathrm{Cb}$, and $u$ be the only cycle vertex in $T^{\prime}$ and $v$ be the $k$-critical vertex in $T^{\prime}[u]$. It is easy to see that all vertices in $T^{\prime}$ except $u$ are descendants of $u$ in $T[r]$. After computing the vertex separation of $T[r]$, each vertex in $T[r]$ obtained a label. The first element in the label of $u$ must be a $k$-critical element and the corresponding $k$-critical vertex is $v$. Since vs $(T[r])=k$, which means vs $(T[r]-T[u])<k$, the first element in the label of $r$ is also a $k$-critical element and the corresponding $k$-critical vertex is $v$, which is not a cycle vertex.

$(\Leftarrow)$. Let $v$ be the $k$-critical vertex in $T[r]$ and let $T^{\prime}$ be the constituent tree that contains $v$ and let $u$ be the only cycle vertex in $T^{\prime}$. It is easy to see that $v$ is a descendant of $u$ in $T[r]$. Thus, $T[v]$ is a subgraph of $T^{\prime}$ and $T^{\prime}$ is a $k$-constituent tree of type $\mathrm{Cb}$ in $U$.

The correctness of the modified algorithm follows from the analysis in Sections 4 and 5 in [7]. We now compare the two algorithms. In our main_modified function, if the condition of the while-loop is satisfied, then by Lemma $2.1, U$ has a $k$-constituent tree of type $\mathrm{Cb}$ that contains $v$. Let $T^{\prime}[u]$ be this constituent tree and $u$ be the only cycle vertex in $T^{\prime}[u]$. The first element in the label of $u$ in $T^{\prime}[u]$ must be $k$-critical element. Let $L(r)$ be the label of $r$ in $T[r]$ and $L(u)$ be the label of $u$ in $T^{\prime}[u]$. We can obtain the label of $r$ in $T[r]-T[v]$ and the label of $u$ in $T^{\prime}[u]-T^{\prime}[v]$ by deleting the first element of each label, according to the definition of labels [7]. This work can be done in constant time. However, without choosing a cycle vertex as the root of $T$, their algorithm needs $O(n)$ time to compute these two labels. Function vs_uni in [7] can only invoke itself in Case 1 when $U$ has a $k$-constituent tree of type Cb. Our main_modified function invokes function vs_uni_modified only when the condition of the while-loop is not satisfied. By Lemma 2.1, in this case, $U$ does not have a $k$-constituent tree of type $\mathrm{Cb}$. Thus in Case 1 of vs_uni_modified, the tree must be of type $\mathrm{C}$, and recursion is avoided. In their function vs_reduced_uni, $v s\left(T_{1}\right)$ and $v s\left(T_{2}\right)$ are computed using $O(n)$ time. However, we compute them before invoking vs_reduced_uni_modified. Let $L_{a}, L_{b}, L_{c}$ and $L_{d}$ be the label of $a$ in $T_{1}[a], b$ in $T_{1}[b], c$ in $T_{2}[c]$ and $d$ in $T_{2}[d]$ respectively. All the information needed by vs_reduced_uni_modified is these four labels. While recursion occurs, we can obtain new labels by simply deleting the first elements from the old ones, which requires only constant time. Hence, the time complexity of vs_reduced_uni_modified can be reduced to $\mathrm{O}(1)$ if we do not count the recursive iterations.

We now analyze the running time of our modified algorithm. Since function vs_reduced_uni_modified only ever invokes itself and the depth of the recursion is $O(\log n)$, its running time is $O(\log n)$. In function vs_uni_modified, Case 1 needs $O(n)$; Cases 3, 4.2, 4.3, 5.2 and 5.3 need $O(n)+O(\log n)$; and other cases can be done in $O(1)$. Thus, the running time of vs_uni_modified is $O(n)+O(\log n)$. In the main_modified function, all the work before invoking vs_uni_modified can be done in $O(n)+O(\log n)$. Therefore, the total running time of the modified algorithm is $O(n)$.

Theorem 2.2 For a unicyclic graph $G$, the vertex separation and the optimal layout of $G$ can be computed in linear time.

For a graph $G$, the 2-expansion of $G$ is the graph obtained by replacing each edge of $G$ by a path of length three. By Theorem 2.2 in [6], the search number of $G$ is equal to the vertex separation of the 2-expansion of $G$. From Theorem 2.2, we have the following result.

Corollary 2.3 For a unicyclic graph $G$, the search number of $G$ can be computed in linear time.

\section{3-cycle-disjoint graphs}

The main work of this section is to propose an $O(n)$ time algorithm to compute the search number and the corresponding optimal search strategy of a 3CDG. In this algorithm we extend the labeling method used in [6]. First of all, we introduce some notation and definitions.

\subsection{Notation and definitions}

For two graphs $G$ and $H$, the union of $G$ and $H$, denoted by $G \cup H$, is the graph with vertex set $V(G) \cup V(H)$ and edge set $E(G) \cup E(H)$. If $H$ is a small graph that consists of only one or two edges, we may use $G \cup E(H)$ to represent $G \cup H$.

Recall that a CDG $G$ is a connected graph such that no pair of cycles in $G$ share a vertex. A rooted $C D G$ is a connected CDG with one vertex designated as the root of the graph. Let $G[r]$ be a rooted CDG with 
root $r$. We first define that each vertex of $G[r]$ except $r$ is a descendant of $r$. For any edge $u v$ that is not on a cycle, the graph $G[r]-u v$ has two connected components. If $u=r$ or $u$ and $r$ are in the same component, then we say that $v$ is a child of $u$, and each vertex that is in the same component as $v$ is called a descendant of $u$. Note that there is no parent-child relationship among vertices in the same cycle. If $v$ is the child of $u$, then we orient this edge with the direction from $u$ to $v$ and the oriented edge is denoted by $(u, v)$. For any cycle $v_{1} v_{2} \ldots v_{k} v_{1}$ in $G[r]$, if $v_{1}$ has an incoming edge $\left(u, v_{1}\right)$ in $G[r]$ or $v_{1}=r$, then $v_{1}$ is referred to as the entrance-vertex of the cycle. For any vertex $v$ of $G[r]$, the subgraph induced by $v$ and all its descendant vertices is called the vertex-branch of $v$, denoted by $G[v] . G[r]$ can be considered as a vertex-branch of $r$. For any directed edge $(u, v)$ of $G[r]$, let $G_{v}$ be the connected component of $G[r]-(u, v)$ that contains $v$. The graph $G_{v} \cup\{(u, v)\}$ is called the edge-branch of the directed edge $(u, v)$, denoted by $G[u v]$. We also say that $G[u v]$ is an edge-branch of $u$. Edge-branch is only defined for non-cycle edges since only these edges are oriented.

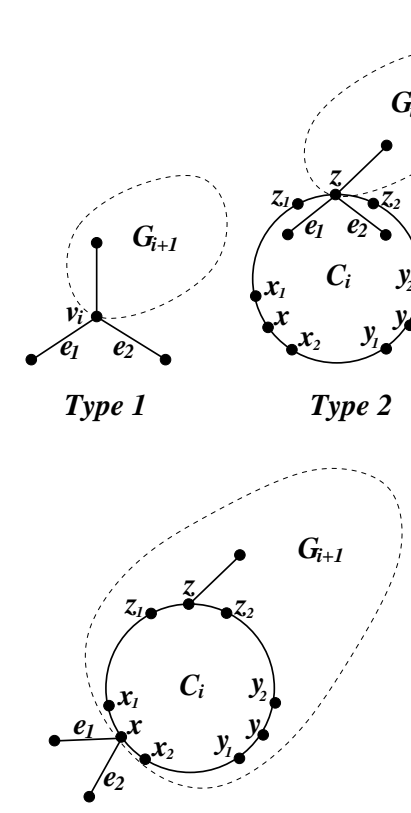

Type 4

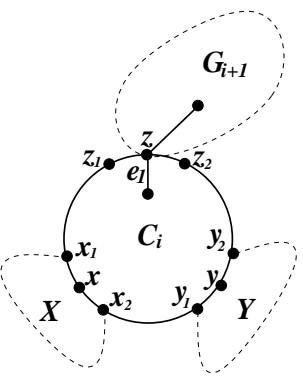

Type 3

Figure 1: five typical critical structures of $G_{i}$

In our labeling process, we assign proper labels to all vertices, non-cycle edges and cycles of a rooted CDG $G[r]$. The label of a vertex $v$, non-cycle edge $e$ or cycle $C$ in $G[r]$ records the necessary structural information of $G[v], G[e]$ or $G[C]$, respectively. Intuitively, a label is a sequence of elements $\left(s_{1}^{t_{1}}, s_{2}^{t_{2}}, \ldots s_{m}^{t_{m}}\right)$, where each element $s_{i}^{t_{i}}$ consists of a positive integer $s_{i}$ and a superscript $t_{i}$, where $0 \leq t_{i} \leq 5$. Let $G_{1}$ be $G[v]$ (similarly, $G[e]$ or $G[C]$ ) and $s_{1}$ be the search number of $G_{1}$. If $G_{1}$ has two edge disjoint subgraphs (they may share a vertex) such that each of them has search number $s\left(G_{1}\right)$, then we say $G_{1}$ is critical and $G_{1}$ must be one of the five typical structures illustrated in Figure 1 and $t_{1}$ indicates which structure it is. If $G_{1}$ is not critical, then we say $G_{1}$ is non-critical and $t_{1}=0$. When $G_{1}$ is critical, according to its type of structure, we can obtain a corresponding reduced graph $G_{2}$ by deleting some vertices from $G_{1}$. $s_{2}$ is the search number of $G_{2}$ and $t_{2}$ indicates the structure of $G_{2}$. Continue this procedure until the reduced graph is non-critical or empty.

Type $1 s\left(G\left[e_{1}\right]\right)=s\left(G\left[e_{2}\right]\right)=s\left(G_{i}\right)$. The reduced graph $G_{i+1}$ is obtained by deleting all the vertices of $G\left[v_{i}\right]$ except $v_{i}$ from $G_{i}$.

Type $2 s\left(G\left[e_{1}\right]\right)=s\left(G\left[e_{2}\right]\right)=s\left(G_{i}\right)$. The reduced graph $G_{i+1}$ is obtained by deleting all the vertices of $G\left[C_{i}\right]$ except $z$ from $G_{i}$.

Type $3 s\left(X \cup Y \cup\left\{x_{2} y_{1}\right\}\right)=s\left(G\left[e_{1}\right]\right)=s\left(G_{i}\right)$. The reduced graph $G_{i+1}$ is obtained by deleting all the vertices of $G\left[C_{i}\right]$ except $z$ from $G_{i}$.

Type $4 s\left(G\left[e_{1}\right]\right)=s\left(G\left[e_{2}\right]\right)=s\left(G_{i}\right)$. The reduced graph $G_{i+1}$ is obtained by deleting all the vertices of $G[x]$ except $x$ from $G_{i}$. 
Type $5 s(X)=s(Y)=s\left(G_{i}\right)$. The reduced graph $G_{i+1}$ is obtained by deleting all the vertices of $G[x]$ except $x$ and all the vertices of $G[y]$ except $y$ from $G_{i}$.

The precise definition of a label is given as follows.

Definition 3.1 Let $G[r]$ be a rooted $3 C D G$, the label of a vertex $v$ (resp. non-cycle edge e or cycle $C$ ) in $G[r]$, denoted by $L(v)$ (resp. $L(e)$ or $L(C)$ ), is defined as a sequence of elements $\left(s_{1}^{t_{1}}, s_{2}^{t_{2}}, \ldots s_{m}^{t_{m}}\right)$. Each element $s_{i}^{t_{i}}$ consists of a positive integer $s_{i}$ and a superscript $t_{i}$, where $0 \leq t_{i} \leq 5$. If $t_{i}=0$, we call $s_{i}^{t_{i}}$ a non-critical element; otherwise, we call it a $s_{i}$-critical element of type- $t_{i}$. The value of $s_{i}^{t_{i}}$, denoted by $\left|s_{i}^{t_{i}}\right|$, is the positive integer $s_{i}$. The value of $L(v)$ (resp. $L(e)$ or $\left.L(C)\right)$, denoted by $|L(v)|(\operatorname{resp} .|L(e)|$ or $|L(C)|)$, is the value of its first element $s_{1}$. $L(v)$ (resp. $L(e)$ or $L(C)$ ) satisfies the following conditions.

1. $s_{1}>s_{2}>\ldots>s_{m}$ and only the last element $s_{m}^{t_{m}}$ can be non-critical.

2. $G_{1}$ is $G[v]$ (resp. $G[e]$ or $G[C]$ ), and for $2 \leq i \leq m, G_{i}$ is defined as a graph obtained from $G_{i-1}$ according to $t_{i-1}$, see condition 3 for details.

3. If $m>1$, then for $i=1,2, \ldots, m-1$, we have $s_{i}=s\left(G_{i}\right), t_{i}>0$ and

(a) if $t_{i}=1$, there exists a non-cycle vertex $v_{i}$ in $G_{i}$ and $v_{i}$ has two outgoing edges $e_{1}$ and $e_{2}$ such that $s\left(G\left[e_{1}\right]\right)=s\left(G\left[e_{2}\right]\right)=s_{i} . G_{i+1}$ is defined as the graph obtained by deleting all the vertices of $G\left[v_{i}\right]$ except $v_{i}$ from $G_{i}$.

(b) if $2 \leq t_{i} \leq 5$, there exists a cycle $C_{i}=z z_{1} x_{1} x x_{2} y_{1} y y_{2} z_{2} z$ (see Figure 1) in $G_{i}$. Let $z$ be the entrance-vertex of $C_{i}$, let $X$ be $G[x] \cup\left\{x x_{1}, x x_{2}\right\}, Y$ be $G[y] \cup\left\{y y_{1}, y y_{2}\right\}$, and $Z$ be $G[z] \cup\left\{z z_{1}, z z_{2}\right\}$. Assume $s(X) \geq s(Y)$, then we have

- if $t_{i}=2, z$ has two outgoing edges $e_{1}$ and $e_{2}$ such that $s\left(G\left[e_{1}\right]\right)=s\left(G\left[e_{2}\right]\right)=s_{i} . G_{i+1}$ is defined as the graph obtained by deleting all the vertices of $G\left[C_{i}\right]$ except $z$ from $G_{i}$.

- if $t_{i}=3, s\left(X \cup Y \cup\left\{x_{2} y_{1}\right\}\right)=s_{i}$ and $z$ has one outgoing edges $e_{1}$ such that $s\left(G\left[e_{1}\right]\right)=s_{i}$. $G_{i+1}$ is defined as the graph obtained by deleting all the vertices of $G\left[C_{i}\right]$ except $z$ from $G_{i}$.

- if $t_{i}=4, x$ has two outgoing edges $e_{1}$ and $e_{2}$ such that $s\left(G\left[e_{1}\right]\right)=s\left(G\left[e_{2}\right]\right)=s_{i} . G_{i+1}$ is defined as the graph obtained by deleting all the vertices of $G[x]$ except $x$ from $G_{i}$.

- if $t_{i}=5, s(X)=s(Y)=s_{i} . G_{i+1}$ is defined as the graph obtained by deleting all the vertices of $G[x]$ except $x$ and all the vertices of $G[y]$ except $y$ from $G_{i}$.

4. $s\left(G_{m}\right)=s_{m}, 0 \leq t_{m} \leq 3$ and

(a) if $t_{m}=1, L$ must be the label of a vertex $v$, and $v$ has two outgoing edges $e_{1}$ and $e_{2}$ such that $s\left(G\left[e_{1}\right]\right)=s\left(G\left[e_{2}\right]\right)=s_{m}$.

(b) if $2 \leq t_{m} \leq 3, L$ must be the label of a cycle $C=z z_{1} x_{1} x x_{2} y_{1} y y_{2} z_{2} z$ (see Figure 1 ). Let $z$ be the entrance-vertex of $C$, let $X$ be $G[x] \cup\left\{x x_{1}, x x_{2}\right\}, Y$ be $G[y] \cup\left\{y y_{1}, y y_{2}\right\}$, and $Z$ be $G[z] \cup\left\{z z_{1}, z z_{2}\right\}$. Assume $s(X) \geq s(Y)$, then we have

- if $t_{i}=2$, $z$ has two outgoing edges $e_{1}$ and $e_{2}$ such that $s\left(G\left[e_{1}\right]\right)=s\left(G\left[e_{2}\right]\right)=s_{m}$.

- if $t_{i}=3, s\left(X \cup Y \cup\left\{x_{2} y_{1}\right\}\right)=s_{m}$ and $z$ has one outgoing edges $e_{1}$ such that $s\left(G\left[e_{1}\right]\right)=s_{m}$.

For the first element $s_{1}^{t_{1}}$ of $L(v)$ (resp. $L(e)$ or $L(C)$ ), if $t_{1}>0, G[v]$ (resp. $G[e]$ or $G[C]$ ) is said to be $s_{1}$-critical of type- $t_{1}$, and the corresponding vertex $v_{1}$ or cycle $C_{1}$ is called the $s_{1}$-critical vertex or $s_{1}$-critical cycle in $G[v]$ (resp. $G[e]$ or $G[C]$ ).

Let $S$ be a monotonic search strategy for a graph $G$ and $v$ be a vertex in $G$. During the procedure of performing $S$ on $G$, if a searcher is placed on $v$ and this searcher is never removed from $v$ until $G$ is cleared, then we say that $S$ ends at $v$; if a searcher is placed on $v$ in the first action of $S$ and this searcher will never been removed from $v$ until all the edges incident with $v$ are cleared, then we say that $S$ starts from $v$. If there is no optimal monotonic search strategy to clear $G$ starting from or ending at $v$, then we call $v$ a $b a d$ vertex of $G$.

For a graph $G$, let $S$ be a search strategy of $G$ that is represented by a sequence of actions, i.e., $S=$ $\left(a_{1}, \ldots, a_{k}\right)$. The reversal of $S$, denoted by $S^{R}$, is defined as $S^{R}=\left(\bar{a}_{k}, \bar{a}_{k-1}, \ldots, \bar{a}_{1}\right)$, where each $\bar{a}_{i}, 1 \leq i \leq k$, is the converse of $a_{i}$, which is defined as follows: the action "place a searcher on vertex $v$ " and the action "remove a searcher from vertex $v$ " are converse with each other; and the action "slide the searcher from $v$ to $u$ along the edge $v u$ " and the action "slide the searcher from $u$ to $v$ along the edge $u v$ " are converse with each other. It is easy to verify that if a search strategy $S$ starts from a vertex $v$, then $S^{R}$ ends at $v$. $S^{R}$ also has the following property. 
Lemma 3.2 If $S$ is an optimal monotonic search strategy of a graph $G$, then $S^{R}$ is also an optimal monotonic search strategy of $G$.

For two sequences of elements $A=\left(a_{1}, \ldots, a_{k}\right)$ and $B=\left(b_{1}, \ldots, b_{m}\right)$. The concatenation of $A$ and $B$, denoted by $A \circ B$, is defined as $A \circ B=\left(a_{1}, \ldots, a_{k}, b_{1}, \ldots, b_{m}\right)$.

For the sake of simplicity, we will use a normalized $3 \mathrm{CDG}$ as the input of our algorithm. For each cycle $C$ in a $3 \mathrm{CDG}$, let $x, y$ and $z$ be the three vertices with degree more than 2. Note that there may not be three vertices each of which has degree more than 2 and in this case, we will choose the degree-two cycle vertex. Recall that there are at least 3 vertices in each cycle since we require that all the graphs in this paper are finite without loops and multiple edges. Replace each of $x \sim y, y \sim z$ and $z \sim x$ by a path of length three such that $C=z z_{1} x_{1} x x_{2} y_{1} y y_{2} z_{2} z$ (see Figure 2). This procedure takes $O(n)$ time. Notice that the search number of the normalized $3 \mathrm{CDG}$ equals the search number of the original graph.

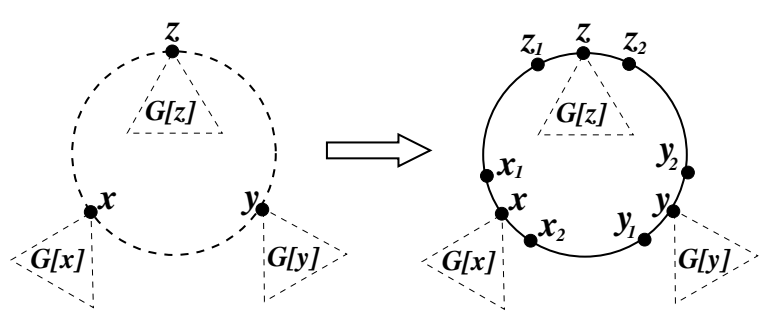

Figure 2: normalization of 3CDG

\subsection{The main idea of the algorithm}

The following algorithm SEARChNumber-3CDG computes the labels of vertices, non-cycle edges and cycles in a rooted 3CDG $G[r]$ by the labeling method. And later we will construct the corresponding optimal search strategy based on these labels.

\footnotetext{
Algorithm SearchNumber-3CDG $(G[r])$

Input: A rooted $3 \mathrm{CDG} G[r]$.

Output: Labels of all vertices, cycles and non-cycle edges.

1. Assign label $\left(0^{0}\right)$ to each leaf (except $r$ if $r$ is also a leaf), $\left(1^{0}\right)$ to each pendant edge and $\left(2^{0}\right)$ to each pendant cycle in $G[r]$.

2. If $r$ is labeled, then return labels of all vertices, cycles and non-cycle edges in $G[r]$.

3. $\quad$ - For each vertex $v$ whose all out-going edges have been labeled, compute the label $L(v)$.

- For each cycle $C$ in which all the vertices with degree more than two have been labeled, compute the label $L(C)$.

- For each non-cycle edge $(u, v)$, if $v$ is on a labeled cycle or if $v$ is a labeled non-cycle vertex, then compute the label $L(u v)$.

- Go to Step 2.
}

It is easy to verify that the label of a pendant edge is $\left(1^{0}\right)$ and the label of a pendant cycle is $\left(2^{0}\right)$. Next we will introduce: (i) how to compute the label of a vertex if all its out-going edges have been labeled; (ii) how to compute the label of a cycle if all the three cycle vertices with degree more than 2 have been labeled; and (iii) how to compute the label of a non-cycle edge if its head is on a labeled cycle or its head is a labeled non-cycle vertex.

\subsection{Computing the label of a vertex}

Lemma 3.3 [2] Let $H$ be a subgraph of $G$ with $s(H)=k$. When we perform an optimal search strategy on $G$, there must exist a vertex $h$ in $H$ such that there are at least $k$ searchers in $H$ after placing a searcher on $h$.

Lemma 3.4 Let $G$ be a graph containing three connected subgraphs $G_{1}, G_{2}$ and $G_{3}$, whose vertex sets are pairwise disjoint, such that for every pair $G_{i}$ and $G_{j}$ there exists a path in $G$ between $G_{i}$ and $G_{j}$ that contains no vertex in the third subgraph. If $s\left(G_{1}\right)=s\left(G_{2}\right)=s\left(G_{3}\right)=k$, then $s(G) \geq k+1$.

Proof. Assume that $s(G)=k$ and let $S$ be an arbitrary monotonic search strategy to clear $G$ using $k$ searchers. Let $h_{i}, 1 \leq i \leq 3$, be the vertex in $G_{i}$ described in Lemma 3.3. W.l.o.g, let $h_{1}, h_{2}, h_{3}$ be the 
order in which $S$ places searchers on them. Since $G_{1}, G_{2}$ and $G_{3}$ are pairwise vertex-disjoint and there is a path between $h_{1}$ and $h_{3}$ containing no vertex of $G_{2}$. At the moment after a searcher is placed on $h_{2}$, all $k$ searchers are in $G_{2}$ and there is no searcher can be used to protect the path between $h_{1}$ and $h_{3}$ from recontamination, which contradicts our initial assumption.

We now consider how to compute the label of a vertex $v$ when the labels of all its outgoing edges are known. Suppose $v$ has $d$ children, $v_{1}, \ldots, v_{d}$. For $1 \leq i \leq d$, let $L\left(v v_{i}\right)$ be the label of $\left(v, v_{i}\right)$ that contains $m_{i}$ elements. For $1 \leq j \leq m_{i}, s_{j, i}^{t_{j, i}}$ is the $j$-th element in $L\left(v v_{i}\right)$. Here we use additional subscripts in $s_{j}$ and $t_{j}$ to indicate whose label it belongs to, i.e., $L\left(v v_{i}\right)=\left(s_{1, i}^{t_{1, i}}, s_{2, i}^{t_{2, i}}, \ldots s_{m_{i}, i}^{t_{m_{i}, i}}\right)$. Then we have a graph $G_{m_{i}, i}$ that is defined in Definition 3.1, where $G\left[v v_{i}\right]$ and $G_{m_{i}, i}$ correspond to $G_{1}$ and $G_{m_{i}}$ respectively. Let $G_{0}$ be the union of all $G_{m_{i}, i}$, for $1 \leq i \leq d$. Let $L_{0}$ be a multiset that contains all non-critical elements of each $L\left(v v_{i}\right)$, $1 \leq i \leq d$. Let $p$ be the value of the largest elements in $L_{0}$ and $q$ be the number of elements with value $p$ in $L_{0}$. We first compute the label of $v$ in $G_{0}[v]$, denoted by $L_{v}$. From Lemma 3.4, we have the following results.

(i) If $q \geq 3$, then $L_{v}=\left((p+1)^{0}\right)$. We can clear $G_{0}$ by $p+1$ searchers ending at $v$ using the following strategy. Place a searcher on $v$ and use other $p$ searchers to clear all the edge-branches of $v$ one by one since each of them has search number at most $p$.

(ii) If $q=2$, then $L_{v}=\left(p^{1}\right)$ and $v$ is the $p$-critical vertex in $G_{0}[v]$. We can clear $G_{0}$ by $p$ searchers using the following strategy. Let $X$ and $Y$ be the two edge-branches with search number $p$. First, clear $X$ with $p$ searchers ending at $v$. Then keep one searcher on $v$ and use other $p-1$ searchers to clear all the other edge-branches of $v$ except $Y$ since each of them has search number at most $p-1$. Finally, clear $Y$ with all $p$ searchers starting from $v$.

(iii) If $q=1$, then $L_{v}=\left(p^{0}\right)$. We can clear $G_{0}$ by $p$ searchers ending at $v$ using the following strategy. Let $X$ be the edge-branch with search number $p$. First, clear $X$ with $p$ searchers ending at $v$. Then keep one searcher on $v$ and use other $p-1$ searchers to clear all the other edge-branches of $v$ since each of them has search number at most $p-1$.

After obtaining the label $L_{v}$ of $v$ in $G_{0}[v]$, we merge $L_{v}$ with all critical elements of $L\left(v v_{i}\right)$, for $1 \leq i \leq d$, which can be done by function MergeLabel-Vertex. The output of this function is the label of $v$ in $G[r]$.

Function MergeLabeL-Vertex $\left(L_{1}, L_{2}, \ldots, L_{d}, L_{v}\right)$

Input: $L_{1}, L_{2}, \ldots, L_{d}$ and $L_{v}$, where $L_{i}$ is the label of the $i$-th outgoing edge of $v$, for $1 \leq i \leq d$, and $L_{v}$ is the label of $v$ in $G_{0}[v]$ that contains only one element.

Output: The label of $v$ in $G[r]$.

1. Set $\alpha \leftarrow$ the only element of $L_{v}$.

2. Let $w$ be the value of the largest repeated critical elements in the labels $L_{1}, L_{2}, \ldots, L_{d}$. ${ }^{*}$ Two critical elements are repeated if they have the same value */

3. if $|\alpha|<w+1$, then $\alpha=(w+1)^{0}$.

4. Let $L$ be a sequence containing all the critical elements of $L_{1}, L_{2}, \ldots, L_{d}$ with value larger than or equal to $|\alpha|$.

5. Set $h \leftarrow$ the value of the last element in $L$;

if $h>|\alpha|$ then return $L(v) \circ(\alpha)$

else update $L$ by deleting its last element; Set $\alpha \leftarrow(|\alpha|+1)^{0}$;

Go to Step 5 .

\subsection{Computing the label of a cycle}

Lemma 3.5 Given a rooted $3 C D G G[r]$ that contains a cycle $C=z z_{1} x_{1} x x_{2} y_{1} y y_{2} z_{2} z$ (see Figure 2). G[x], $G[y]$ and $G[z]$ are the vertex-branches of $x, y$ and $z$ respectively. Let $X$ be $G[x] \cup\left\{x x_{1}, x x_{2}\right\}, Y$ be $G[y] \cup$ $\left\{y y_{1}, y y_{2}\right\}$, and $Z$ be $G[z] \cup\left\{z z_{1}, z z_{2}\right\}$. If $s(X)=s(Y)=k, s(Z) \leq k$ and neither $X[x]$ nor $Y[y]$ is $k$-critical, then $s(G)=k+1$ if and only if $s(Z)=k$.

Proof. $(\Leftarrow)$. If $s(X)=s(Y)=s(Z)=k$, it follows from Lemma 3.4 that $s(G) \geq k+1$. We now show that $G$ can be cleared by $k+1$ searchers starting from $z$. We know that $s(G[z]) \leq k$ since $G[z]$ is a subgraph of $Z$. We first station one searcher on $z$ and use $k$ searchers to clear $G[z]$; then slide one searcher from $z$ along the path $z z_{2} y_{2} y$ to $y$; slide the searcher stationed on $z$ along the path $z z_{1} x_{1} x$ to $x$; use one searcher to clear the path $x x_{2} y_{1} y$; use $k$ searchers to clear $G[x]$ starting from $x$ since $G[x]$ is not $k$-critical; finally, use $k$ searchers to clear $G[y]$ starting from $y$ since $G[y]$ is not $k$-critical either. Thus, $G$ can be cleared by using $k+1$ searchers starting from $z$. 
$(\Rightarrow)$. Suppose $s(Z)<k$. We show that $G$ can be cleared by $k$ searchers. Let $S$ be an optimal search strategy of $Z$. W.l.o.g., let us assume the edge $z z_{1}$ is cleared before $z z_{1}$ in $S$. First we clear $G[x]$ by $k$ searchers ending at $x$; station one searcher on $x$ and then perform $S$ on $Z$. During this procedure, when we use one searcher to clear the edge $z z_{1}$, we clear the path $z z_{1} x_{1} x$ instead and then slide the searcher on $x$ along the path $x x_{2} y_{1} y$ to $y$; when we use one searcher to clear the edge $z z_{2}$, we clear the path $z z_{2} y_{2} y$ instead. After $S$ is finished, $X$ and $Z$ and $C$ are all cleared. Then we can use $k$ searchers to clear $G[y]$ starting from $y$ since $G[y]$ is not k-critical.

Lemma 3.6 Given a rooted 3CDG G[r] that contains a cycle $C=z z_{1} x_{1} x x_{2} y_{1} y y_{2} z_{2} z$ (see Figure 2). If $s(G[x])=k$ and $x$ has two outgoing edges $e_{1}$ and $e_{2}$ such that $s\left(G\left[e_{1}\right]\right)=s\left(G\left[e_{2}\right]\right)=k$ and none of these two edge-branches is $k$-critical. Let $G^{*}$ be the graph obtained from $G$ by deleting $G[x]$ (including $x$ ). Then $s(G) \geq k+1$ if and only if $s\left(G^{*}\right) \geq k$.

Proof. $(\Rightarrow)$. Suppose $s\left(G^{*}\right)<k$. We show that $G$ can be cleared by $k$ searchers. First, we use $k$ searchers to clear $G\left[e_{1}\right]$ ending at $x$; keep one searcher on $x$ and clear all the other edge-branches of $x$ except $G\left[e_{2}\right]$ using other $k-1$ searchers since each of them has search number at most $k-1$; clear $G^{*}$ and edges $x_{1} x$ and $x_{2} x$ using $k-1$ searchers since $s\left(G^{*}\right)<k$; finally, clear $G\left[e_{2}\right]$ starting from $x$ using $k$ searchers.

$(\Leftarrow)$. If $s\left(G^{*}\right) \geq k$, it follows from Lemma 3.4 that $s(G) \geq k+1$.

We now consider how to compute the label of a cycle $C$ when we know the labels of all the three cycle vertices with degree more than 2 .

Let $C=z z_{1} x_{1} x x_{2} y_{1} y y_{2} z_{2} z$ be a cycle in a rooted $3 \mathrm{CDG} G[r]$ and $z$ be the entrance-vertex of $C$. Suppose $L(x), L(y)$ and $L(z)$ are the labels of $x, y$ and $z$ respectively. Let $X, Y$ and $Z$ be the graphs defined as in Lemma 3.5. By using function MergeLabel-Vertex to merge $L(x)$ (resp. $L(y)$ or $L(z)$ ) with $\left(1^{1}\right)$, we can obtain the label of $x$ (resp. $y$ or $z$ ) in $X[x]$ (resp. $Y[y]$ or $Z[z]$ ), denoted by $L_{x}$ (resp. $L_{y}$ or $L_{z}$ ). $L_{x}=\left(s_{1, x}^{t_{1, x}}, s_{2, x}^{t_{2, x}}, \ldots s_{m_{x}, x}^{t_{m_{x}, x}}\right)$. Then we have a graph $G_{m_{x}, x}$ that is defined in Definition 3.1, where $X[x]$ and $G_{m_{x}, x}$ correspond to $G_{1}$ and $G_{m_{x}}$ respectively. Similarly, we have $G_{m_{y}, y}$ and $G_{m_{z}, z}$. For simplicity, we use $X^{\prime}, Y^{\prime}, Z^{\prime}$ to denote $G_{m_{x}, x}, G_{m_{y}, y}, G_{m_{z}, z}$ respectively and $a_{X}, a_{Y}, a_{Z}$ to denote $s_{m_{x}, x}^{t_{m_{x}, x}}, s_{m_{y}, y}^{t_{m_{y}, y}}, s_{m_{z}, z}^{t_{m_{z}, z}}$ respectively. Let $p$ be the largest value among $a_{X}, a_{Y}$ and $a_{Z}$. Let $G_{0}=X^{\prime} \cup Y^{\prime} \cup Z^{\prime} \cup C$. The following cases decide the label of the cycle $C$ in $G_{0}[z]$, denoted by $L_{C}$.

CASE 1: More than one of $a_{X}, a_{Y}$ and $a_{Z}$ are $p$-critical.

$L_{C}=\left((p+1)^{0}\right)$. By Lemma 3.4, $s\left(G_{0}\right) \geq p+1$ and we can use $p+1$ searchers to clear $G_{0}$ ending at vertex $z$ by following strategy. First, we station one searcher on vertex $x$; clear $X^{\prime}$ using $p$ searchers since it has search number at most $p$. $y$ has at most two edge-branches with search number $p$ and none of them is $p$-critical. We clear one of the largest edge-branches of $y$ using $p$ searchers ending at $y$; use one searcher to clear the path $x x_{2} y_{1} y$; slide the searcher on $x$ from $x$ to $z$ along the path $x x_{1} z_{1} z$ and keep it on $z$; use one searcher to clear the path $z z_{2} y_{2} y$; clear all the other edge-branches of $y$ except the second largest one using $p-1$ searchers since each of them has search number at most $p-1$; use $p$ searchers to clear the second largest edge-branch of $y$ starting from $y$; finally, clear $Z^{\prime}$ using $p$ searchers since it has search number at most $p$.

CASE 2: Only one of $a_{X}, a_{Y}$ and $a_{Z}$ is $p$-critical.

CASE 2.1: $a_{Z}$ is $p$-critical. Let $G^{*}=X^{\prime} \cup Y^{\prime} \cup\left\{x_{2} y_{1}\right\}$.

CASE 2.1.1: $s\left(G^{*}\right)<p$.

$L_{C}=\left(p^{2}\right)$. By Lemma 3.6, $s\left(G_{0}\right)=p$ and we can clear $G_{0}$ using $p$ searchers.

CASE 2.1.2: $s\left(G^{*}\right) \geq p$.

$L_{C}=\left((p+1)^{0}\right)$. By Lemma 3.6, $s\left(G_{0}\right) \geq p+1$. We can use $p+1$ searchers to clear $G_{0}$ ending at vertex $z$ by a similar strategy described in CASE 1 .

CASE 2.2: $a_{X}\left(\right.$ or $\left.a_{Y}\right)$ is $p$-critical. Let $G^{*}=Z^{\prime} \cup Y^{\prime} \cup\left\{y_{2} z_{2}\right\}$ (or $G^{*}=Z^{\prime} \cup X^{\prime} \cup\left\{z_{1} x_{1}\right\}$ ).

CASE 2.2.1: $s\left(G^{*}\right)<p$.

Let $L$ be the label of $z$ in $G^{*}[z] . L_{C}=\left(p^{4}\right) \circ L$. By Lemma 3.6, $s\left(G_{0}\right)=p$ and we can clear $G_{0}$ by $p$ searchers.

CASE 2.2.2: $s\left(G^{*}\right) \geq p$.

$L_{C}=\left((p+1)^{0}\right)$. By Lemma 3.6, $s\left(G_{0}\right) \geq p+1$. We can clear $G_{0}$ by $p+1$ searchers ending at vertex $z$ using a similar strategy described in CASE 1 .

CASE 3: None of $a_{X}, a_{Y}$ and $a_{Z}$ is $p$-critical. 
CASE 3.1: $a_{X}=a_{Y}=a_{Z}=p$.

$L_{C}=\left((p+1)^{0}\right)$. By Lemma 3.5, $s\left(G_{0}\right) \geq p+1$. We can clear $G_{0}$ by $p+1$ searchers ending at vertex $z$ using a similar strategy described in CASE 1 .

CASE 3.2: Exactly two of $a_{X}, a_{Y}$ and $a_{Z}$ have value $p$.

CASE 3.2.1: $a_{X}=a_{Y}=p$.

Let $L$ be the label of $z$ in $Z^{\prime}[z] . L_{C}=\left(p^{5}\right) \circ L$. By Lemma $3.5, s\left(G_{0}\right)=p$ and we can clear $G_{0}$ using $p$ searchers.

CASE 3.2.2: $a_{Z}=p$ and $\left(a_{X}=p\right.$ or $\left.a_{Y}=p\right)$.

CASE 3.2.2.1: One edge-branch of $z$ in $Z^{\prime}[z]$ has search number $p$. $L_{C}=\left(p^{3}\right)$. By Lemma 3.6, $s\left(G_{0}\right)=p$ and we can clear $G_{0}$ using $p$ searchers.

CASE 3.2.2.2: No edge-branch of $z$ in $Z^{\prime}[z]$ has search number $p$.

$L_{C}=\left(p^{0}\right)$. By Lemma 3.6, $s\left(G_{0}\right)=p$ and we can clear $G_{0}$ by $p$ searchers ending at $z$ by the following strategy. W.l.o.g, assume $\left|a_{X}\right|=p$. First, we use $p$ searchers to clear $X^{\prime}$ ending at $x . y$ has at most two edge-branches with search number $p-1$ and none of them is $(p-1)$-critical. We clear one of the largest edge-branches of $y$ using $p-1$ searchers ending at $y$; use one searcher to clear the path $x x_{2} y_{1} y$; slide the searcher on $x$ from $x$ to $z$ along the path $x x_{1} z_{1} z$ and keep it on $z$; use one searcher to clear the path $z z_{2} y_{2} y$; clear all the other edge-branches of $y$ except the second largest one using $p-2$ searchers since each of them has search number at most $p-2$; use $p-1$ searchers to clear the second largest edge-branch of $y$ starting from $y$; finally, clear the edge-branches of $z$ using $p-1$ searchers since each of them has search number at most $p-1$.

CASE 3.3: Only one of $a_{X}, a_{Y}$ and $a_{Z}$ has value $p$.

CASE 3.3.1: $a_{Z}=p$ and there is one edge-branch of $z$ in $Z^{\prime}[z]$ with search number $p$. Let $G^{*}=X^{\prime} \cup Y^{\prime} \cup$ $\left\{x_{2} y_{1}\right\}$.

CASE 3.3.1.1: $s\left(G^{*}\right)=p$.

$L_{C}=\left(p^{3}\right)$. We can clear $G_{0}$ by $p$ searchers using the following strategy. First, use $p$ searchers to clear $X^{\prime}$ ending at $x . y$ has at most two edge-branches with search number $p-1$ and none of them is $(p-1)$-critical. We clear one of the largest edge-branches of $y$ using $p-1$ searchers ending at $y$; use one searcher to clear the path $x x_{2} y_{1} y$; silde the searcher on $x$ from $x$ to $z$ along the path $x x_{1} z_{1} z$ and keep it on $z$; use one searcher to clear the path $z z_{2} y_{2} y$; clear all the other edge-branches of $y$ except the second largest one using $p-2$ searchers since each of them has search number at most $p-2$; use $p-1$ searchers to clear the second largest edge-branch of $y$ starting from $y$; finally, we clear $Z^{\prime}$ using $p$ searchers starting from $z$.

CASE 3.3.1.2: $s\left(G^{*}\right)<p$.

$L_{C}=\left(p^{0}\right)$. We can clear $G_{0}$ using $p$ searchers ending at vertex $z$ using the following strategy. First, use $p$ searchers to clear the largest edge-branch of $z$ ending at $z$ and then keep one searcher on $z$; use $p-1$ searchers to clear all the other edge-branches of $z$ since each of them has search number less than $p$; finally, use $p-1$ searchers to clear $G^{*}$.

CASE 3.3.2: $a_{Z}=p$ and no edge-branch of $z$ in $Z^{\prime}[z]$ has search number $p$.

$L_{C}=\left(p^{0}\right)$. We can use $p$ searchers to clear $G_{0}$ ending at vertex $z$ by a similar strategy described in CASE 3.2.2.2.

CASE 3.3.3: $a_{X}=p$ or $a_{Y}=p$.

$L_{C}=\left(p^{0}\right)$. We can use $p$ searchers to clear $G_{0}$ ending at vertex $z$ by a similar strategy described in CASE 3.2.2.2.

After obtaining the label $L_{C}$ of $C$ in $G_{0}[z]$, we will use the following function MERGELABEL-CyCLE to merge $L_{C}$ with the three labels $L_{x}-a_{X}, L_{y}-a_{Y}$ and $L_{z}-a_{Z}$. The output of this function is the label of $C$ in $G[r]$.

Function MergeLabel-Cycle $\left(L_{x}, L_{y}, L_{z}, L_{C}\right)$

Input: $L_{x}, L_{y}, L_{z}$ and $L_{C}$, where $L_{x}\left(\right.$ resp. $L_{y}$ or $\left.L_{z}\right)$ is the label of $x$ (resp. $y$ or $z$ ) in $X[x]$ (resp. $Y[y]$ or $Z[z]$ ) without the last element and $L_{C}$ is the label of $C$ in $G_{0}[z]$. Output: The label of $C$ in $G[r]$.

1. Set $\alpha \leftarrow$ the first element of $L_{C}, q \leftarrow\left|L_{C}\right|$.

2. Let $w$ be the value of the largest repeated critical element of the input labels $L_{x}, L_{y}$ and $L_{z}$. $/^{*}$ Two critical elements are repeated if they have the same value */

3. if $|\alpha|<w+1$, then $\alpha=(w+1)^{0}$. 
4. Let $L$ be a sequence containing all the critical elements of $L_{x}, L_{y}$ and $L_{z}$ with value larger than or equal to $|\alpha|$.

5. Set $h \leftarrow$ the value of the last element in $L$;

if $h>|\alpha|$ then if $|\alpha|=q$ then return $L(v) \circ L_{C}$

else return $L(v) \circ(\alpha)$;

else update $L$ by deleting its last element;

Set $\alpha \leftarrow(|\alpha|+1)^{0}$;

Go to Step 5 .

\subsection{Computing the label of a non-cycle edge}

Let $G[r]$ be a rooted $3 \mathrm{CDG}$ and $(u, v)$ be a non-cycle edge in $G[r]$. If $v$ is on a labeled cycle or $v$ is a labeled non-cycle vertex, then function EDGELABEL computes the label of $(u, v)$ in $G[r]$, denoted by $L(u v)$.

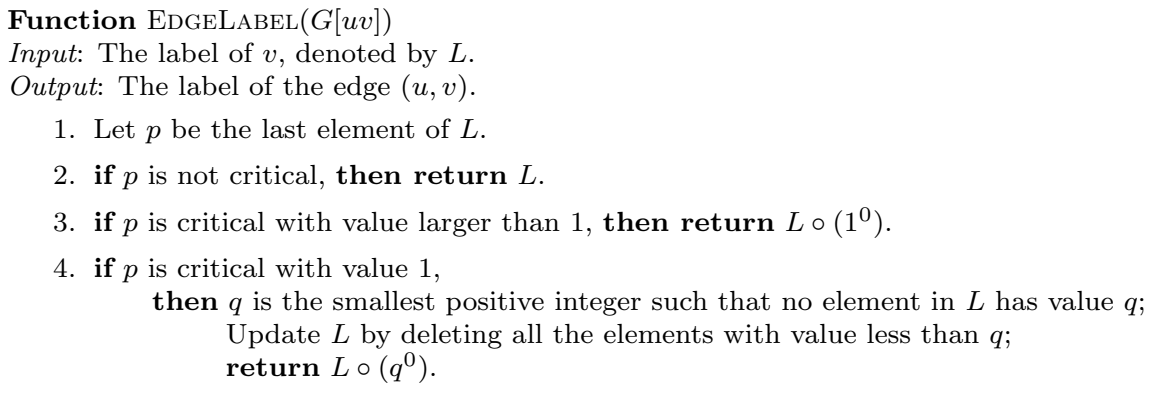

\subsection{Correctness and time complexity}

Lemma 3.7 Function MERGeLABEL-VERTEX outputs the label of a vertex in $G[r]$.

Proof. Let $G[r]$ be a rooted $3 \mathrm{CDG}$ and $v$ be a vertex in $G[r]$. Let $v_{1}, \ldots, v_{d}$ be the $d$ children of $v$. For $1 \leq i \leq d$, let $L_{i}=\left(s_{1, i}^{t_{1, i}}, s_{2, i}^{t_{2, i}}, \ldots s_{m_{i}, i}^{t_{m_{i}, i}}\right)$ be the label of $\left(v, v_{i}\right)$ in $G[r]$. From Definition 3.1, the last element of each $L_{i}$ is non-critical. Let $L_{v}$ be the label of $v$ in $G_{0}[v]$, which is defined in Section 3.3. The input of function MERGELABEL-VERTEX is $L_{1}, L_{2}, \ldots, L_{d}$ and $L_{v}$.

Line 1-2: Recall that $\alpha$ is the only element of $L_{v}$ and $w$ is the value of the largest repeated critical elements among all elements in $L_{1}, L_{2}, \ldots, L_{d}$.

Line 3: There two cases regarding the value of $|\alpha|$.

1. $|\alpha|<w+1$. For each $i, 1 \leq i \leq d$, we can find the index $j$ such that in $L_{i},\left|s_{j-1, i}^{t_{j-1, i}}\right|>w \geq\left|s_{j, i}^{t_{j, i}}\right|$ (if $w \geq\left|s_{1, i}^{t_{1, i}}\right|$, then $j=1$ ). Then we have a graph $G_{j, i}$ that is defined in Definition 3.1, where $G\left[v v_{i}\right]$ and $G_{j, i}$ correspond to $G_{1}$ and $G_{j}$ respectively. Let $G^{\prime}$ be the union of all $G_{j, i}, 1 \leq i \leq d$. Since $w$ is the value of the largest repeated critical elements, there are at least two disjoint $w$-critical branches in $G^{\prime}$. By Lemma 3.4, $s\left(G^{\prime}\right) \geq w+1$. And $G^{\prime}$ can be cleared by $w+1$ searchers starting from $v$ : station one searcher on $v$ and use other $w$ searchers to clear every edge-branch of $v$ since it has search number at most $w$. Thus $s\left(G^{\prime}\right)=w+1$ and update $\alpha=(w+1)^{0}$.

2. $|\alpha| \geq w+1$. For each $i, 1 \leq i \leq d$, we can find the index $j$ such that in $L_{i},\left|s_{j-1, i}^{t_{j-1, i}}\right| \geq|\alpha|>\left|s_{j, i}^{t_{j, i}}\right|$ (if $\left|s_{m_{i}, i}^{t_{m_{i}, i}}\right| \geq|\alpha|$, then $\left.j=m_{i}\right)$. Then we have a graph $G_{j, i}$ that is defined in Definition 3.1, where $G\left[v v_{i}\right]$ and $G_{j, i}$ correspond to $G_{1}$ and $G_{j}$ respectively. It is easy to see that each $G_{j, i}$ has search number less than or equal to $|\alpha|$ and if its search number equals $|\alpha|$, then it is not $|\alpha|$-critical. Let $G^{\prime}$ be the union of all $G_{j, i}, 1 \leq i \leq d$. First, we know $s\left(G^{\prime}\right) \geq|\alpha|$ since $G^{\prime}$ contains $G_{0}$ as its subgraph and $s\left(G_{0}\right)=|\alpha|$. Then, notice that at most two of $G_{j, i}$ have search number equal to $|\alpha|$ and $G^{\prime}$ can be cleared by $|\alpha|$ searchers. So $s\left(G^{\prime}\right)=|\alpha|$.

After Line 3, we have $s\left(G^{\prime}\right)=|\alpha|$ and $v$ has no $|\alpha|$-critical edge-branch in $G^{\prime}[v]$ ( $v$ may have one $|\alpha|$-critical vertex-branch and in such case, $v$ must be the $|\alpha|$-critical vertex).

Line 4: Let $L$ be the sequence formed by all the critical elements of the input labels $L_{1}, L_{2}, \ldots, L_{d}$ with value larger than or equal to $|\alpha|$. Notice that $L$ contains no repeated element.

Line 5: For each $i, 1 \leq i \leq d$, we can find the index $j$ such that in $L_{i},\left|s_{j-1, i}^{t_{j-1, i}}\right|>|\alpha| \geq\left|s_{j, i}^{t_{j, i}}\right|\left(\right.$ if $|\alpha| \geq\left|s_{1, i}^{t_{1, i}}\right|$, then $j=1$ ). Then we have a graph $G_{j, i}$ that is defined in Definition 3.1, where $G\left[v v_{i}\right]$ and $G_{j, i}$ correspond to $G_{1}$ and $G_{j}$ respectively. Let $G^{\prime \prime}$ be the union of all $G_{j, i}, 1 \leq i \leq d$. If the last element of $L$ is $|\alpha|$-critical, which means a certain $G_{j, i}$ is $|\alpha|$-critical. We have $s\left(G^{\prime}\right)=|\alpha|$, where $G^{\prime}$ is defined in Line 3 . $G^{\prime}$ and the $|\alpha|$-critical $G_{j, i}$ are edge disjoint. Thus, we have $s\left(G^{\prime \prime}\right) \geq|\alpha|+1$. It is easy to clear $G^{\prime \prime}$ using $|\alpha|+1$ searchers ending at $v$. Update $\alpha=(|\alpha|+1)^{0}$ and update $L$ by deleting the last element. Repeat this step until the 
last element of $L$ has value larger than $|\alpha|$. At this time, $L \circ(\alpha)$ satisfies the definition of the label of $v$ in $G[r]$.

Based on Lemma 3.7 and the discussion in Section 3.4, we have the following lemma.

Lemma 3.8 Function MERGELABEL-CyClE outputs the label of a cycle in $G[r]$.

Lemma 3.9 Function EDGELABEL outputs the label of a non-cycle edge in $G[r]$.

Proof. Let $G[r]$ be a rooted $3 \mathrm{CDG}$, and $(u, v)$ be a non-cycle edge in $G[r]$ where $v$ is on a labeled cycle or $v$ is a labeled non-cycle vertex. Note that the label of $(u, v)$ should be the same as the label of $u$ if $G[u v]$ is the only one edge-branch at $u$. In that case, $G[u]$ has only one more edge $(u, v)$ than $G[v]$ (resp. $G[C]$ if $v$ is on a labeled cycle $C$ ). The work done by function EDGELABEL is to merge the label of $v($ resp. $C$ ) with a label $\left(1^{0}\right)$ that is the label of a single edge. Thus, by Lemma 3.7, function EDGELABEL outputs the label of $(u, v)$ in $G[r]$.

From Lemmas 3.7, 3.8 and 3.9, SEARCHNumber-3CDG can compute the labels of each vertex, cycle and non-cycle edge. In the rest of this section we will analyze the time complexity of this algorithm.

We introduce a data structure used in [6] that compresses the label representation. For a sub-list of value consecutive critical elements in a label, we use an interval to represent them. For example, the label $\left(9^{t_{1}}, 8^{t_{2}}, 7^{t_{3}}, 6^{t_{4}}, 5^{t_{5}}, 3^{t_{6}}, 2^{t_{7}}, 1^{0}\right)$ is represented as $\left((9,5),(3,2), 1^{0}\right)$. Note that the non-critical element is not put into any interval. The benefit of this representation is to improve the label merging operation. For example, if we want to merge label $\left(9^{t_{1}}, 8^{t_{2}}, 7^{t_{3}}, 6^{t_{4}}, 5^{t_{5}}, 3^{t_{6}}, 2^{t_{7}}, 1^{0}\right)$ with $\left(5^{0}\right)$, we can obtain the result $\left(10^{0}\right)$ in one step by using this compressed representation.

Lemma 3.10 The time complexity of function EDGELABEL is $O(1)$ with the compressed label representation.

Proof. If the input label $L$ is in the compressed label representation, we alter Lines 2,3 and 4 of the function as follows.

2. if the last element of $L$ is non-critical, then return $L$.

3. if the last element of $L$ is $(x, y)$ and $x \geq y>1$, then return $L \circ\left(1^{0}\right)$.

4. if the last element of $L$ is $(x, 1)$ and $x \geq 1$, then return $(L-(x, 1)) \circ\left((x+1)^{0}\right)$.

It is easy to verify that these operations are equivalent to the original ones and each operation takes constant time.

Lemma 3.11 The time complexity of function MERGELABEL-VERTEX is $O\left(\left|L_{2}\right|+d\right)$, where $L_{2}$ is the second largest label among $L_{1}, L_{2}, \ldots, L_{d}$.

Proof. Lines 1 and 3 take constant time. Line 5 also takes $O(1)$ time by using the same technique as in function EdGeLABEL. We now consider the time complexity of Lines 2 and 4 .

For $1 \leq i \leq d$, suppose that $\left|L_{1}\right| \geq\left|L_{2}\right| \geq\left|L_{i}\right|$ for $3 \leq i \leq d$. In order to achieve $O\left(\left|L_{2}\right|+d\right)$ time for MERGeLABEL-VERTEX, we first merge part of $L_{1}$ with all the other labels and then merge the result with the rest of $L_{1}$. Replace Line 2 by the following fragment.

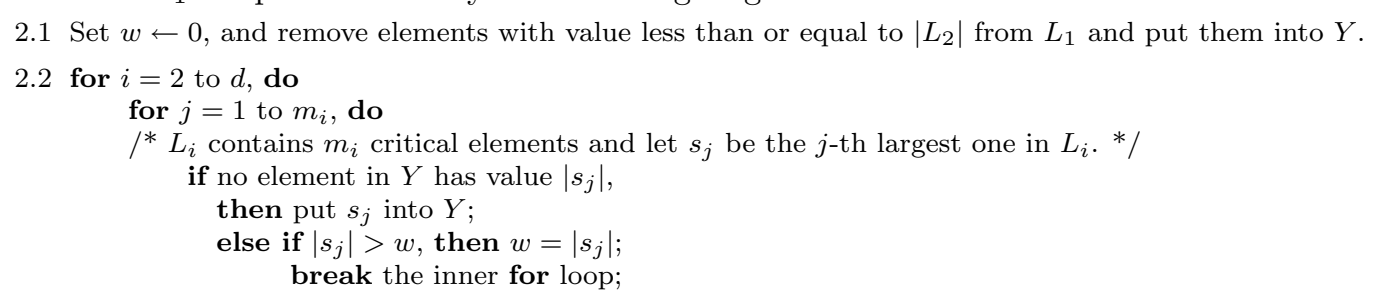

2.3 Delete all elements with value less than or equal to $w$ from $Y$.

2.4 Represent $Y$ by the compressed form.

Line 2.1 takes $O\left(\left|L_{2}\right|\right)$ time. In Line 2.2, each time when we check an element, we either add it into $Y$ or finish checking the label that contains it. The size of $Y$ is at most $\left|L_{2}\right|$ and there are $d$ labels. Thus, Line 2.2 takes $O\left(\left|L_{2}\right|+d\right)$ time and Lines 2.3 and 2.4 take $O\left(\left|L_{2}\right|\right)$ time. Hence, the time complexity of Line 2 is $O\left(\left|L_{2}\right|+d\right)$.

After the execution of this fragment, $w$ is the value of the largest repeated critical elements in $L_{1}, L_{2}, \ldots, L_{d}$ and $Y$ contains all the critical elements with value less than or equal to $\left|L_{2}\right|$ and larger than $w$ (if there is no such element, $Y$ is empty). Consider the following two cases regarding the value of $\alpha$ after Line 3 .

CASE 1. $|\alpha|>\left|L_{2}\right|+1$. Then critical elements with value larger than or equal to $|\alpha|$ can only appear in $L_{1}$. Let $L$ be $L_{1}$ and Line 4 takes $O(1)$ time. 
CASE 2: $|\alpha| \leq\left|L_{2}\right|+1$. Let $L=L_{1} \circ Y$ and delete all elements in $L$ with value smaller than $|\alpha|$. Line 4 takes $O\left(\left|L_{2}\right|\right)$ time.

Therefore, the time complexity of function MergeLabel-Vertex is $O\left(\left|L_{2}\right|+d\right)$.

Similarly to Lemma 3.11, we have the following lemma.

Lemma 3.12 The time complexity of function MERGELABEL-CyClE is $O(\beta)$, where $\beta$ is the value of the second largest label among $L_{x}, L_{y}$ and $L_{z}$.

Lemma 3.13 If a function $f(n)$ is defined on the positive integers by the recurrence equation

$$
f(n)= \begin{cases}c, & n=1,2 \\ f\left(m_{1}\right)+c, & k=1, n \geq 3 \\ \max _{M}\left\{\sum_{i=1}^{k} f\left(m_{i}\right)+c\left(\left\lceil\log m_{2}\right\rceil+k\right)\right\}, & k \geq 2, n \geq 3\end{cases}
$$

where $M=\left\{\left(m_{1}, m_{2}, \ldots, m_{k}\right): m_{1} \geq m_{2} \geq \cdots m_{k} \geq 1\right.$, and $\left.\sum_{i=1}^{k} m_{i}=n-2\right\}$, and $c \geq 1$ is a constant, then $f(n)$ is $O(n)$.

Proof. We use induction to show that $f(n) \leq c(3 n-\lceil\log n\rceil-1)$. It is easy to verify that this is true for $n=1,2$. Assume the inequality is true for any $n \leq N-1$. Now let us consider $n=N$.

Case 1: $k=1$.

$$
\begin{aligned}
f(N) & =f\left(m_{1}\right)+c \\
& =f(N-1)+c \\
& \leq c(3(N-1)-\lceil\log (N-1)\rceil-1)+c \\
& =c(3 N-(2+\lceil\log (N-1)\rceil)-1) \\
& \leq c(3 N-\lceil\log N\rceil-1) .
\end{aligned}
$$

Case 2: $k \geq 2$.

Let $k_{1}=\left|\left\{m_{i} \mid m_{i} \geq 2,1 \leq i \leq k\right\}\right|$ and $k_{2}=\left|\left\{m_{i} \mid m_{i}=1,1 \leq i \leq k\right\}\right|$. We have that $k_{1}+k_{2}=k$ and $\sum_{i=k_{1}+1}^{k} f\left(m_{i}\right)=c \cdot k_{2}$.

$$
\begin{aligned}
f(N)= & \max \left\{\sum_{i=1}^{k} f\left(m_{i}\right)+c\left(\left\lceil\log m_{2}\right\rceil+k\right)\right\} \\
= & \max \left\{\sum_{i=1}^{k_{1}} f\left(m_{i}\right)+\sum_{i=k_{1}+1}^{k} f\left(m_{i}\right)+c\left(\left\lceil\log m_{2}\right\rceil+k\right)\right\} \\
= & \max \left\{\sum_{i=1}^{k_{1}} f\left(m_{i}\right)+c\left(k_{2}+\left\lceil\log m_{2}\right\rceil+k\right)\right\} \\
\leq & \max \left\{c\left(3 \sum_{i=1}^{k_{1}} m_{i}-\sum_{i=1}^{k_{1}}\left\lceil\log m_{i}\right\rceil-k_{1}\right)+c\left(k_{2}+\left\lceil\log m_{2}\right\rceil+k\right)\right\} \\
= & \max \left\{c\left(3\left(N-1-k_{2}\right)-k_{1}+k_{2}+k-\left\lceil\log m_{1}\right\rceil-\sum_{i=3}^{k_{1}}\left\lceil\log m_{i}\right\rceil\right)\right\} \\
= & \max \left\{c\left(3 N-\left(2+k_{2}+\sum_{i=3}^{k_{1}}\left\lceil\log m_{i}\right\rceil+\left\lceil\log m_{1}\right\rceil\right)-1\right)\right\} \\
& \left(\operatorname{note} \text { that } \sum_{i=3}^{k}\left\lceil\log m_{i}\right\rceil=0 \text { when } k=2\right) \\
\leq & c(3 N-\lceil\log N\rceil-1) .
\end{aligned}
$$

Let $\Delta=2+k_{2}+\sum_{i=3}^{k_{1}}\left\lceil\log m_{i}\right\rceil+\left\lceil\log m_{1}\right\rceil$. Now we show that $\Delta \geq\lceil\log N\rceil$. Case $2.1: k_{1}=0$. We have that $k=k_{2}=N-1$ and $m_{1}=1$. In this case, $\Delta=1+N \geq\lceil\log N\rceil$. Case 2.2: $1 \leq k_{1} \leq 2$. We have that $k=k_{1}+k_{2} \leq 2+k_{2}$. In this case, $\Delta=2+k_{2}+\left\lceil\log m_{1}\right\rceil \geq k+\left\lceil\log m_{1}\right\rceil \geq\lceil\log N\rceil$. Case 2.3: $k_{1} \geq 3$. For $3 \leq i \leq k_{1}$, we have that $\left\lceil\log m_{i}\right\rceil \geq 1$ since $m_{i} \geq 2$. In this case, $\Delta=2+k_{2}+\sum_{i=3}^{k_{1}}\left\lceil\log m_{i}\right\rceil+\left\lceil\log m_{1}\right\rceil \geq$ $2+k_{2}+k_{1}-2+\left\lceil\log m_{1}\right\rceil=k+\left\lceil\log m_{1}\right\rceil \geq\lceil\log N\rceil$ 
In algorithm SEARCHNumber-3CDG, we use $f(G[v])$ to denote the time used to compute the label of vertex $v$ and use $f(G[C])$ to denote the time used to compute the label of cycle $C$. Then we have

$$
f(G[v])=f\left(G\left[v v_{1}\right]\right)+f\left(G\left[v v_{2}\right]\right)+\cdots+f\left(G\left[v v_{d}\right]\right)+O\left(s\left(G\left[v v_{2}\right]\right)+d\right),
$$

where $v$ has $d$ edge-branches and $G\left[v v_{2}\right]$ is the second largest edge-branch according to their search numbers.

$$
f(G[C])=f(G[x])+f(G[y])+f(G[z])+O\left(s\left(G^{*}\right)\right),
$$

where $x, y$ and $z$ are the three vertices on $C$ with degree more than 2 , and $G^{*}$ is one of $G[x], G[y]$ and $G[z]$ that has the second largest search number.

The search number of a tree is $O(\log n)$, where $n$ is the number of vertices in that tree. And from Theorem 5.1, the search number of a $3 C D G$ is also $O(\log n)$. Theorem 3.14 follows from Lemma 3.13.

Theorem 3.14 For a rooted 3CDG G[r], algorithm SEARChNumber-3CDG computes the labels of all vertices, cycles and non-cycle edges in $O(n)$ time.

\subsection{Constructing an optimal search strategy}

Theorem 3.15 Let $G[r]$ be a rooted 3CDG. If the labels of all vertices, cycles and non-cycle edges are known, then we can construct an optimal search strategy for $G$ in $O(n)$ time.

PROOF. The following algorithm SEARCH3CDG constructs an optimal search strategy of a rooted 3CDG.

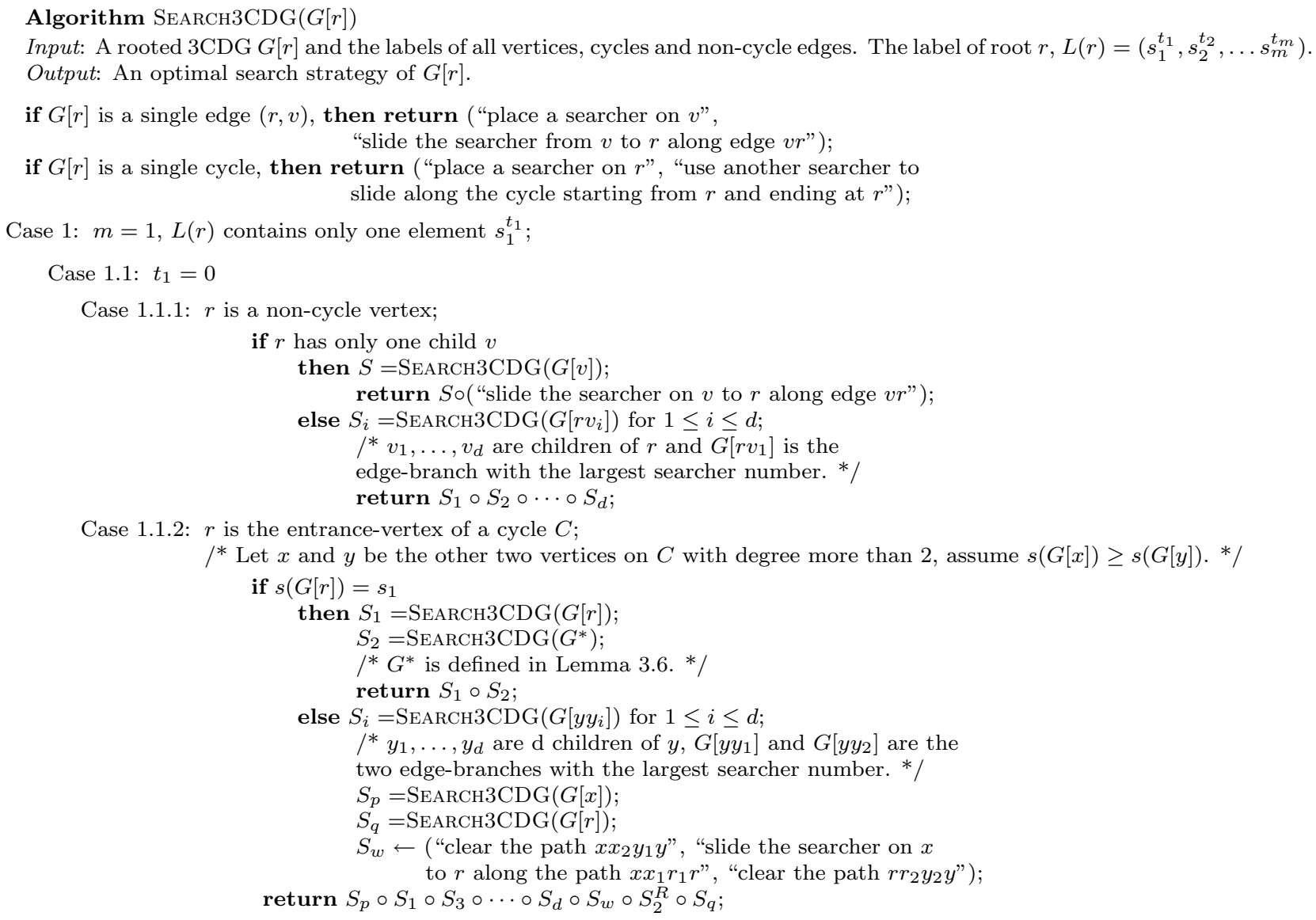




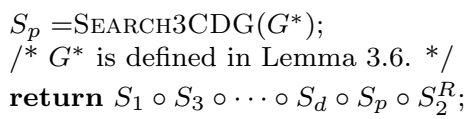

Case 1.4: $t_{1}=3$

$S_{i}=\operatorname{SeARCH} 3 \mathrm{CDG}\left(G\left[y y_{i}\right]\right)$ for $1 \leq i \leq d$;

$/^{*} y_{1}, \ldots, y_{d}$ are children of $y$ and $G\left[y y_{1}\right]$ and $G\left[y y_{2}\right]$ are two edge-branches with the largest searcher number.

$* /$

$S_{p}=\operatorname{SEARCH} 3 \mathrm{CDG}(G[x])$

$S_{q}=\operatorname{SEARCH} 3 \mathrm{CDG}(G[r])$;

$S_{w} \leftarrow$ ("clear the path $x x_{2} y_{1} y$ ", "slide the searcher on $x$ to $z$ along the path $x x_{1} z_{1} z$ ", "clear the path $z z_{2} y_{2} y$ ");

return $S_{p} \circ S_{1} \circ S_{3} \circ \cdots \circ S_{d} \circ S_{w} \circ S_{2}^{R} \circ S_{q}^{R}$

Case 2: $m>1, L(r)$ contains more than one element;

$/^{*}$ Let $G[r]$ (or $G[C]$ if $r$ is on cycle $C$ ) be $G_{1}$, and $G_{2}$ be defined in Definition 3.1.

Let $x, y$ and $z$ be the three vertices on $C_{1}$ with degree more than 2 and $z$ be the entrance-vertex of $C_{1}$. $* /$

Case 2.1: $t_{1}=1$

$S_{i}=\operatorname{SEARCH} 3 \mathrm{CDG}\left(G\left[v_{1} u_{i}\right]\right)$ for $1 \leq i \leq d$;

$/^{*} u_{1}, \ldots, u_{d}$ are children of $v_{1}$ and $G\left[v_{1} u_{1}\right]$ and $G\left[v_{1} u_{2}\right]$ are two edge-branches with searcher number $s_{1}$. ${ }^{*} /$

$S_{p}=\operatorname{SeARCH} 3 \mathrm{CDG}\left(G_{2}\right)$;

return $S_{1} \circ S_{3} \circ \cdots \circ S_{d} \circ S_{p} \circ S_{2}^{R}$

Case 2.2: $t_{1}=2$

$S_{i}=\operatorname{SEARCH} 3 \mathrm{CDG}\left(G\left[z z_{i}\right]\right)$ for $1 \leq i \leq d$

${ }^{*} z_{1}, \ldots, z_{d}$ are children of $z$ and $G\left[z z_{1}\right]$ and $G\left[z z_{2}\right]$ are two edge-branches with searcher number $s_{1}$. */

$S_{p}=\operatorname{SEARCH} 3 \mathrm{CDG}\left(G_{2}\right)$;

$S_{q}=\operatorname{SEARCH} 3 \mathrm{CDG}\left(G^{*}\right)$;

/* $G^{*}$ is defined in Lemma 3.6.*/

return $S_{1} \circ S_{3} \circ \cdots \circ S_{d} \circ S_{p} \circ S_{q} \circ S_{2}^{R}$

Case 2.3: $t_{1}=3$

$S_{i}=\operatorname{SeARCH} 3 \mathrm{CDG}\left(G\left[y y_{i}\right]\right)$ for $1 \leq i \leq d$

$/^{*} y_{1}, \ldots, y_{d}$ are children of $y$ and $G\left[\bar{y} y_{1}\right]$ and $G\left[y y_{2}\right]$ are two edge-branches with the largest searcher number.

*/

$S_{p}=\operatorname{SEARCH} 3 \mathrm{CDG}(G[x])$;

$S_{q}=\operatorname{SeARCH} 3 \mathrm{CDG}(G[z])$;

$S_{h}=\operatorname{SEARCH} 3 \mathrm{CDG}\left(G_{2}\right)$;

$S_{w} \leftarrow$ ("clear the path $x x_{2} y_{1} y$ ", "slide the searcher on $x$ to $z$ along the path $x x_{1} z_{1} z$ ", "clear the path $z z_{2} y_{2} y$ ");

return $S_{p} \circ S_{1} \circ S_{3} \circ \cdots \circ S_{d} \circ S_{w} \circ S_{2}^{R} \circ S_{h} \circ S_{q}^{R}$;

Case 2.4: $t_{1}=4$

$S_{i}=\mathrm{SEARCH} 3 \mathrm{CDG}\left(G\left[x x_{i}\right]\right)$ for $1 \leq i \leq d$

$/^{*} x_{1}, \ldots, x_{d}$ are children of $x$ and $G\left[x x_{1}\right]$ and $G\left[x x_{2}\right]$ are two edge-branches with searcher number $s_{1}$. */

$S_{p}=\operatorname{SEARCH} 3 \mathrm{CDG}\left(G_{2}\right)$;

return $S_{1} \circ S_{3} \circ \cdots \circ S_{d} \circ S_{p} \circ S_{2}^{R}$

Case 2.5: $t_{1}=5$

$S_{1}=\operatorname{SeARCH} 3 \mathrm{CDG}(G[x]) ;$

$S_{2}=\operatorname{SeARCH} 3 \mathrm{CDG}(G[y])$

$S_{3}=\operatorname{Search} 3 \mathrm{CDG}\left(G_{2}\right)$;

Modify $S_{3}$ by inserting the action "slide the searcher on $x$ to $y$ along path $x x_{2} y_{1} y$ " after the action by which edge $x_{1} x$ is cleared;

return $S_{1} \circ S_{3} \circ S_{2}^{R}$;

The correctness of this algorithm follows from our discussion in Sections 3.3, 3.4 and 3.5. Notice that if $L(r)$ contains only one element and this element is non-critical, algorithm SEARCH3CDG will return an optimal search strategy of $G[r]$ ending at $r$.

We now analyze the running time of this algorithm. The work done by this algorithm outside the recursive calls take $O(1)$ time. Each time when the algorithm invokes itself, the input 3CDG will be divided into several non-empty edge disjoint subgraphs. Since a 3CDG has $O(n)$ edges, where $n$ is the number of vertices, this algorithm invokes itself for at most $O(n)$ times. Therefore, the total running time of SEARCH3CDG is $O(n)$. 


\section{$4 \quad k$-ary cycle-disjoint graphs}

A complete $k$-ary tree $T$ is a rooted $k$-ary tree in which all leaves have the same depth and every internal vertex has $k$ children. If we replace each vertex of $T$ with a $(k+1)$-cycle such that each vertex of internal cycle has degree at most 3 , then we obtain a cycle-disjoint graph $G$, which we call a $k$-ary cycle-disjoint graph (k-ary $C D G$ ). In $T$, we define the level of the root be 1 and the level of a leaf be the number of vertices in the path from the root to that leaf. We use $T_{k}^{h}$ to denote a complete $k$-ary tree with level $h$ and $G_{k}^{h}$ to denote the $k$-ary CDG obtained from $T_{k}^{h}$ (see Figure 3).

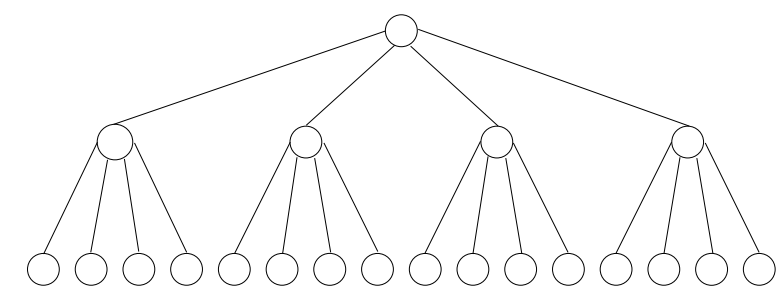

Figure 3: $G_{4}^{3}$, a 4-ary CDG with level 3

In this section, we will show how to compute the search numbers of $k$-ary CDGs. Similar to [7], we have the following lemmas.

Lemma 4.1 For a connected graph $G$, let $C=v_{1} v_{2} \ldots v_{m} v_{1}$ be a cycle in $G$ such that each $v_{i}(1 \leq i \leq m)$ connects to a connected subgraph $X_{i}$ by a bridge. If $s\left(X_{i}\right) \leq k, 1 \leq i \leq m$, then $s(G) \leq k+2$.

Lemma 4.2 For a connected graph $G$, let $v_{1}, v_{2}, v_{3}, v_{4}$ and $v_{5}$ be five vertices on a cycle $C$ in $G$ such that each $v_{i}(1 \leq i \leq 5)$ connects to a connected subgraph $X_{i}$ by a bridge. If $s\left(X_{i}\right) \geq k, 1 \leq i \leq 5$, then $s(G) \geq k+2$.

Lemma 4.3 For a connected graph $G$, let $C=v_{1} v_{2} v_{3} v_{4} v_{1}$ be a 4-cycle in $G$ such that each $v_{i}(1 \leq i \leq 4)$ connects to a connected subgraph $X_{i}$ by a bridge. If $s(G)=k+1$ and $s\left(X_{i}\right)=k, 1 \leq i \leq 4$, then for any optimal search strategy of $G$, the first cleared vertex and the last cleared vertex must be in two distinct graphs $X_{i}+v_{i}, 1 \leq i \leq 4$.

Lemma 4.4 For a $C D G G$ with search number $k$, let $S$ be an optimal monotonic search strategy of $G$ in which the first cleared vertex is a and the last cleared vertex is $b$. If there are two cut-vertices $a^{\prime}$ and $b^{\prime}$ in $G$ such that an edge-branch $G_{a^{\prime}}$ of $a^{\prime}$ contains a and an edge-branch $G_{b^{\prime}}$ of $b^{\prime}$ contains $b$ and the graph $G^{\prime}$ obtained by removing $G_{a^{\prime}}$ and $G_{b^{\prime}}$ from $G$ is connected, then we can use $k$ searchers to clear $G^{\prime}$ starting from $a^{\prime}$ and ending at $b^{\prime}$.

Lemma 4.5 Let $G$ be a connected graph and $C$ be a cycle of length at least four in $G$, and $v_{1}$ and $v_{2}$ be two vertices of $C$ such that each $v_{i}(1 \leq i \leq 2)$ connects to a connected subgraph $X_{i}$ by a bridge $v_{i} v_{i}^{\prime}$. If $s\left(X_{1}\right)=s\left(X_{2}\right)=k$ and $v_{1}^{\prime}$ is a bad vertex of $X_{1}$ or $v_{2}^{\prime}$ is a bad vertex of $X_{2}$, then we need at least $k+2$ searchers to clear $G$ starting from $v_{3}$ and ending at $v_{4}$, where $v_{3}$ and $v_{4}$ are any two vertices on $C$ other than $v_{1}$ and $v_{2}$.

Lemma 4.6 For a connected graph $G$, let $v_{1}, v_{2}, v_{3}$ and $v_{4}$ be four vertices on a cycle $C$ in $G$ such that each $v_{i}(1 \leq i \leq 4)$ connects to a connected subgraph $X_{i}$ by a bridge $v_{i} v_{i}^{\prime}$. If $s\left(X_{i}\right)=k$, and $v_{i}^{\prime}$ is a bad vertex of $X_{i}, 1 \leq i \leq 4$, then $s(G) \geq k+2$.

From the above lemmas, we can prove the major result of this section.

Theorem 4.7 Let $T_{k}^{h}$ be a complete k-ary tree with level $h$ and $G_{k}^{h}$ be the corresponding k-ary CDG.

(i) If $k=2$ and $h \geq 3$, then $s\left(T_{2}^{h}\right)=\left\lfloor\frac{h}{2}\right\rfloor+1$ and $s\left(G_{2}^{h}\right)=\left\lfloor\frac{h}{2}\right\rfloor+2$.

(ii) If $k=3$ and $h \geq 2$, then $s\left(T_{3}^{h}\right)=h$ and $s\left(G_{3}^{h}\right)=h+1$.

(iii) If $k=4$ and $h \geq 2$, then $s\left(T_{4}^{h}\right)=h$ and $s\left(G_{4}^{h}\right)=h+\left\lceil\frac{h}{2}\right\rceil$.

(iv) If $k \geq 5$ and $h \geq 2$, then $s\left(T_{k}^{h}\right)=h$ and $s\left(G_{k}^{h}\right)=2 h$. 
Proof. The search numbers of complete $k$-ary trees can be verified directly by SEARCHNUMBER-3CDG since a tree can be regarded as a $3 \mathrm{CDG}$ without any cycle. Thus, we will only consider the search numbers of $k$-ary CDGs.

(i) The search number of $G_{2}^{h}$ can be verified directly by SEARCHNUMBER-3CDG since $G_{2}^{h}$ are 3CDGs.

(ii) We now prove $s\left(G_{3}^{h}\right)=h+1$ by induction on $h$. Let $R=r_{0} r_{1} r_{2} r_{3} r_{0}$ be the cycle in $G_{3}^{h}$ that corresponds to the root of $T_{3}^{h}$. Suppose $r_{0}$ is the vertex without any outgoing edges. When $h=2$, it is easy to see that $s\left(G_{3}^{2}\right)=3$ and all four vertices of $R$ are not bad vertices in $G_{3}^{2}$. Suppose $s\left(G_{3}^{h}\right)=h+1$ holds when $h<n$ and all four vertices of $R$ are not bad vertices in $G_{3}^{h}$. When $h=n, R$ has three edge-branches with search number $n$. It follows from Lemma 3.4 that $s\left(G_{3}^{n}\right) \geq n+1$. We will show how to use $n+1$ searchers to clear the graph by the following strategy: use $n$ searchers to clear $G\left[r_{1}\right]$ ending at $r_{1}$; keep one searcher on $r_{1}$ and use $n$ searchers to clear $G\left[r_{2}\right]$ ending at $r_{2}$; use one searcher to clear the edge $r_{1} r_{2}$; slide the searcher on $r_{1}$ to $r_{0}$ and slide the searcher on $r_{2}$ to $r_{3}$; use one searcher to clear the edge $r_{0} r_{3}$; then clear $G\left[r_{3}\right]$ with $n$ searchers starting from $r_{3}$. This strategy never needs more than $n+1$ searchers. Thus, $s\left(G_{3}^{n}\right)=n+1$. From this strategy, it is easy to see that all four vertices of $R$ are not bad vertices in $G_{3}^{n}$.

(iii) We will prove $s\left(G_{4}^{h}\right)=h+\left\lceil\frac{h}{2}\right\rceil$ by induction on $h$. Let $R=r_{0} r_{1} r_{2} r_{3} r_{4} r_{0}$ be the cycle in $G_{4}^{h}$ that corresponds to the root of $T_{4}^{h}$. Suppose $r_{0}$ is the vertex without any outgoing edges. We want to show that if $h$ is odd, then no bad vertex is on $R$, and if $h$ is even, then $r_{0}$ is a bad vertex of $G_{4}^{h}$.

When $h=2$, it is easy to see that $s\left(G_{4}^{2}\right)=3$ and $r_{0}$ is a bad vertex in $G_{4}^{2}$. When $h=3$, by Lemma 4.6 , $s\left(G_{4}^{3}\right) \geq 5$ and it is easy to verify that 5 searchers can clear $G_{4}^{3}$ starting from any one of the five vertices on $R$. Suppose these results hold for $G_{4}^{h}$ when $h<n$. We now consider the two cases when $h=n$.

If $n$ is odd, $G\left[r_{i}\right]$ has search number $n-1+(n-1) / 2$ and $r_{i}$ is a bad vertex in $G\left[r_{i}\right], 1 \leq i \leq 4$. By Lemma 4.6, we have $s\left(G_{4}^{n}\right) \geq n-1+(n-1) / 2+2=n+(n+1) / 2$. We will show how to use $n+(n+1) / 2$ searchers to clear the graph by the following strategy. Let $v$ be any one of the cycle vertex of $R$. We first place two searchers $\alpha$ and $\beta$ on $v$ and then slide $\beta$ along $R$ starting from $v$ and ending at $v$. Each time when $\beta$ arrives a vertex of $R$, we clear the subgraph attached to this vertex using $n-1+(n-1) / 2$ searchers. This strategy never needs more than $n+(n+1) / 2$ searchers. Thus, $s\left(G_{4}^{n}\right)=n+(n+1) / 2$. It is also easy to see that all five vertices of $R$ are not bad vertices in $G_{4}^{n}$.

If $n$ is even, $G\left[r_{i}\right]$ has search number $n-1+n / 2$ and $r_{i}$ is not a bad vertex in $G\left[r_{i}\right], 1 \leq i \leq 4$. By Lemma 3.4, we have $s\left(G_{4}^{n}\right) \geq n+n / 2$. We will show how to use $n+n / 2$ searchers to clear the graph by the following strategy: use $n-1+n / 2$ searchers to clear $G\left[r_{1}\right]$ ending at $r_{1}$; use $n-1+n / 2$ searchers to clear $G\left[r_{2}\right]$ ending at $r_{2}$; use one searcher to clear the edge $r_{1} r_{2}$; slide the searcher on $r_{1}$ along the path $r_{1} r_{0} r_{4}$ to $r_{4}$; slide the searcher on $r_{2}$ to $r_{3}$ along the edge $r_{2} r_{3}$; use one searcher to clear the edge $r_{3} r_{4} ;$ clear $G\left[r_{3}\right]$ with $n-1+n / 2$ searchers starting from $r_{3}$ and finally clear $G\left[r_{4}\right]$ with $n-1+n / 2$ searchers starting from $r_{4}$. This strategy never needs more than $n+n / 2$ searchers. Thus, $s\left(G_{4}^{n}\right)=n+n / 2$ and, by Lemma $4.3, r_{0}$ is a bad vertex in $G_{4}^{n}$.

(iv) The search number of $G_{k}^{h}, k \geq 5$, can be verified directly from Lemmas 4.1 and 4.2.

\section{Approximation algorithms}

Megiddo et al. [12] introduced the concept of the hub and the avenue of a tree. Given a tree $T$ with $s(T)=k$, only one of the following two cases must happen: (1) $T$ has a vertex $v$ such that all edge-branches of $v$ have search number less than $k$, this vertex is called a $h u b$ of $T$; and (2) $T$ has a unique path $v_{1} v_{2} \ldots v_{t}, t>1$, such that $v_{1}$ and $v_{t}$ each has exactly one edge-branch with search number $k$ and each $v_{i}, 1<i<t$, has exactly two edge-branches with search number $k$, this unique path is called an avenue of $T$.

Theorem 5.1 Given a CDG G, if $T$ is a tree obtained by contracting each cycle of $G$ into a vertex, then $s(T) \leq s(G) \leq 2 s(T)$.

Proof. Since $T$ is a minor of $G$, we have $s(T) \leq s(G)$. We prove the second inequality by induction on $s(T)$. If $s(T)=1, T$ is a single vertex or a path, and correspondingly, $G$ is a single vertex or a single cycle or a sequence of cycles connected by paths. It is easy to see that we can clear $G$ using at most 2 searchers. Suppose $s(G) \leq 2 s(T)$ holds for $s(T) \leq k-1, k \geq 2$. When $s(T)=k$, we consider the following two cases.

1. $T$ has a hub $v$. Every edge-branch of $v$ in $T$ has search number at most $k-1$. By induction, the subgraph in $G$ that corresponds to an edge-branch of $v$ has search number at most $2(k-1)$. If $v$ corresponds to a vertex $v^{\prime}$ in $G$, then we can place a searcher on $v^{\prime}$ and use $2(k-1)$ searchers to clear each subgraph attached to $v^{\prime}$. If $v$ corresponds to a cycle $C$ in $G$, then let $v^{\prime}$ be a vertex of $C$. We first place two searchers $\alpha$ and $\beta$ on $v^{\prime}$ and then slide $\beta$ along $C$ starting from $v^{\prime}$ and ending at $v^{\prime}$. Each time when $\beta$ arrives a vertex of $C$, we clear the subgraph attached to this vertex using $2(k-1)$ searchers. 
2. $T$ has an avenue $p$. Every edge-branch attached to $p$ has search number at most $k-1$. By induction, the subgraph in $G$ that corresponds to an edge-branch attached to $p$ has search number at most $2(k-1)$. The subgraph of $G$, say $p^{\prime}$, that corresponds to $p$ is a path or a sequence of cycles connected by paths. In this case, we can use two searchers to clear $p^{\prime}$ while using $2(k-1)$ searchers to clear each subgraph attached to $p^{\prime}$.

Hence, when $s(T)=k, s(G) \leq 2 k$. Therefore, $s(T) \leq s(G) \leq 2 s(T)$. For a $k$-ary CDG $G_{k}^{h}$ and $k \geq 5$, by Theorem 4.7, $s\left(G_{k}^{h}\right)=2 s\left(T_{k}^{h}\right)$, which indicates that the second inequality is tight.

In Theorem 5.1, if $G$ consists of two cycles linked by an edge, then $T$ is an edge. Thus $s(T)=1$ and $s(G)=2$. Hence, the second inequality is tight.

Corollary 5.2 For any $C D G$, there is an $O(n)$ time approximation algorithm with approximation ratio 2.

Proof. Let $G$ be a cycle-disjoint graph and $S_{G}$ be the search strategy described in the proof of Theorem 5.1. Recall that $S_{G}$ is constructed from $S_{T}$ that is the optimal search strategy for $T$, where $T$ is the tree obtained by contracting each cycle of $G$ into a vertex. Since it takes linear time to compute $S_{T}$, it is easy to see that $S_{G}$ can also be found in linear time. Let $\kappa\left(S_{G}\right)$ be the number of searchers required by $S_{G}$. We have

$$
\frac{\kappa\left(S_{G}\right)}{s(G)} \leq \frac{2 s(T)}{s(T)}=2 .
$$

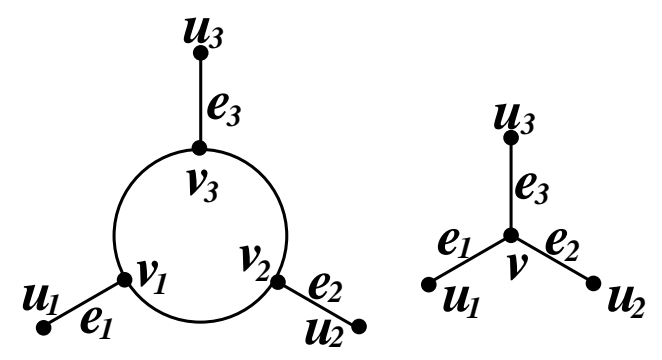

Figure 4: vertices of cycle and the corresponding tree nodes

Lemma 5.3 Let $T$ be the tree obtained from a $3 C D G G$ by contracting every cycle of $G$ into a vertex. If the degree of each cycle vertex in $G$ is at most 3, then $s(G) \leq s(T)+1$.

Proof. Let $s(T)=k$ and let $S_{T}$ be a monotonic search strategy to clear $T$ using $k$ searchers. We will show that $G$ can be cleared using at most $k+1$ searchers by constructing a new search strategy $S_{G}$ for $G$. $S_{G}$ is a subsequence of $S_{T}$ and contains some new actions that clear the cycle edges by an extra searcher $\lambda$.

Initially, let $S_{G}$ be the same as $S_{T}$. Let $C$ be a cycle of $G$ and $v$ be the corresponding vertex of $T$. In $G, v_{i}$ and $u_{i}$ are the two endpoints of $e_{i}, i=1,2,3$. In $T, v$ and $u_{i}$ are the two endpoints of $e_{i}, i=1,2,3$. See Figure 4. W.l.o.g., assume $e_{1}, e_{2}$ and $e_{3}$ of $T$ are cleared in the described order. First, we modify some actions of $S_{G}$ by the following operations: replace the action "place a searcher on $v$ " by "place a searcher on $v_{1}$ "; replace the action "slide a searcher along the edge $u_{i} v$ from $u_{i}$ to $v$ " by "slide a searcher along $u_{i} v_{i}$ from $u_{i}$ to $v_{i}$ ", $i=1,2,3$; replace the action "slide a searcher along the edge $v u_{i}$ from $v$ to $u_{i}$ " by "slide a searcher along $v_{i} u_{i}$ from $v_{i}$ to $u_{i}$ " $, i=1,2,3$; replace the action "remove a searcher from $v$ " by "remove a searcher from $v_{3} "$.

Suppose $e_{2}$ is cleared in the $i^{\text {th }}$ action of $S_{T}$. Then $e_{1}$ is cleared before the $i^{t h}$ action and there is at least one searcher $\alpha$ on $v$ and $\alpha$ remains on $v$ in the $i^{t h}$ action of $S_{T}$ since the edge $e_{3}$ is still contaminated. There are two possible actions to clear $e_{2}$ in $S_{T}$.

Case 1 In $S_{T}$, a searcher $\beta$ slides from $v$ to $u_{2}$ by the $i^{\text {th }}$ action. In this case, there is a corresponding $\beta$ on $v_{1}$ in $S_{G}$ at this moment. In this case, we insert five new actions immediately before the corresponding $i^{t h}$ action in $S_{G}$. "slide $\alpha$ from $v_{1}$ to $v_{3}$ ", "slide $\beta$ from $v_{1}$ to $v_{2}$ ", "place $\lambda$ on $v_{2}$ ", "slide $\lambda$ from $v_{2}$ to $v_{3}$ ", "remove $\lambda$ from $v_{3}$ ".

Case 2 In $S_{T}$, a searcher $\beta$ slides from $u_{2}$ to $v$ by the $i^{\text {th }}$ action. In this case, there is a corresponding $\beta$ on $u_{2}$ at this moment. In this case, we insert five new actions in $S_{G}$ immediately after the corresponding $i^{t h}$ action in $S_{G}$. "place $\lambda$ on $v_{2}$ ", "slide $\lambda$ from $v_{2}$ to $v_{1}$ ", "remove $\lambda$ from $v_{1}$ ", "slide $\alpha$ from $v_{1}$ to $v_{3}$ ", "slide $\beta$ from $v_{2}$ to $v_{3}$ ". 
Each time after we clear $C$ by the new actions added in $S_{G}, \lambda$ is free. It is easy to verify that $S_{G}$ can clear $G$ using no more than $k+1$ searchers.

Lemma 5.4 Given a graph $G$, for any two vertices a and $b$ of $G$, there is a search strategy that uses at most $s(G)+1$ searchers to clear $G$ starting from a and ending at $b$.

Proof. Let $S$ be an optimal search strategy of $G$. It follows from Lemma 3.2 that $S^{R}$ is also an optimal search strategy of $G$. Vertex $a$ is cleared before $b$ either in $S$ or in $S^{R}$. We first place a searcher $\lambda$ on $a$ and keep it on $a$; then perform the search strategy on $G$ in which $a$ is cleared before $b$; at the moment $a$ is cleared, remove $\lambda$ from $a$ and place it on $b$ and keep it on $b$ until $G$ is cleared. Thus, we can clear $G$ starting from $a$ and ending at $b$ with no more than $s(G)+1$ searchers.

Definition 5.5 Let $G$ be a connected graph and $X_{1}, X_{2}, \ldots, X_{m}$ be an edge partition of $G$ such that each $X_{i}$ is a connected subgraph and each pair of $X_{i}$ share at most one vertex. Let $G^{*}$ be a graph of $m$ vertices such that each vertex of $G^{*}$ corresponds to a $X_{i}$ and there is an edge between two vertices of $G^{*}$ if and only if the corresponding two $X_{i}$ share a common vertex.

Theorem 5.6 Let $G, G^{*}$ and $X_{i}, 1 \leq i \leq m$, be defined in Definition 5.5. If $G^{*}$ is a path, then there is a search strategy that uses at most $\max _{1 \leq i \leq m} s\left(X_{i}\right)+1$ searchers to clear $G$.

Proof. Suppose $G^{*}$ is the path $v_{1} v_{2} \ldots v_{m}$ and $v_{i}$ corresponds to $X_{i}, 1 \leq i \leq m$. Let $a_{i}$ be the vertex shared by $X_{i}$ and $X_{i+1}, 1 \leq i \leq m-1$ and let $a_{0}$ be a vertex in $X_{1}$ and $a_{m}$ be a vertex in $X_{m}$. By Lemma 5.4, we can use $s\left(X_{i}\right)+1$ searchers to clear each $X_{i}$ starting from $a_{i-1}$ and ending at $a_{i}$, for $X_{1}, X_{2}, \ldots, X_{m}$. Therefore, there is a search strategy uses at $\operatorname{most}_{\max _{i}} s\left(X_{i}\right)+1$ searchers to clear $G$.

Theorem 5.7 Let $G, G^{*}$ and $X_{i}, 1 \leq i \leq m$, be defined in Definition 5.5. If $G^{*}$ is a tree, then there is a search strategy that uses at most $\max _{1 \leq i \leq m} s\left(X_{i}\right)+\left\lceil\Delta\left(G^{*}\right) / 2\right\rceil s\left(G^{*}\right)$ searchers to clear $G$, where $\Delta\left(G^{*}\right)$ is the maximum degree of $G^{*}$.

Proof. We prove the result by induction on $s\left(G^{*}\right)$. If $s\left(G^{*}\right)=1, G^{*}$ is a single vertex or a path, $\left\lceil\Delta\left(G^{*}\right) / 2\right\rceil=1$, the result can be verified directly from Theorem 5.6. Suppose this result holds for $s\left(G^{*}\right) \leq n$, $n \geq 2$. When $s\left(G^{*}\right)=n+1$, we consider the following two cases.

CAse 1. $G^{*}$ has a hub $v$. Let $X(v)$ be the subgraph of $G$ that corresponds to $v$ and $S$ be an optimal search strategy of $X(v)$. Each subgraph that corresponds to a neighbor of $v$ in $G^{*}$ shares a vertex with $X(v)$ in $G$. Divide these shared vertices into $\lceil\operatorname{deg}(v) / 2\rceil$ pairs such that for each pair of vertices $a_{i}$ and $a_{i}^{\prime}, a_{i}$ is cleared before $a_{i}^{\prime}$ is cleared in $S, 1 \leq i \leq\lceil\operatorname{deg}(v) / 2\rceil$. Let $v_{i}$ (resp. $v_{i}^{\prime}$ ) be the neighbor of $v$ such that its corresponding subgraph of $G$, denoted by $X\left(v_{i}\right)$ (resp. $X\left(v_{i}^{\prime}\right)$ ), shares $a_{i}$ (resp. $\left.a_{i}^{\prime}\right)$ with $X(v)$. Let $v$ be the root of $G^{*}$, let $T_{i}$ (resp. $\left.T_{i}^{\prime}\right)$ be the vertex-branch of $v_{i}$ (resp. $\left.v_{i}^{\prime}\right)$ and let $X\left(T_{i}\right)\left(\operatorname{resp} . X\left(T_{i}^{\prime}\right)\right)$ be the subgraph of $G$ that is the union of the subgraphs that correspond to all vertices in $T_{i}$ (resp. $T_{i}^{\prime}$ ). Obviously $a_{i}$ (resp. $a_{i}^{\prime}$ ) is the only vertex shared by $X(v)$ and $X\left(T_{i}\right)$ (resp. $X\left(T_{i}^{\prime}\right)$ ). Since $v$ is a hub of $G^{*}$, we know that $s\left(T_{i}\right) \leq n$. Thus, $s\left(X\left(T_{i}\right)\right) \leq \max _{i} s\left(X_{i}\right)+\left\lceil\Delta\left(T_{i}\right) / 2\right\rceil n \leq \max _{i} s\left(X_{i}\right)+\left\lceil\Delta\left(G^{*}\right) / 2\right\rceil n$. First, we place a searcher on each $a_{i}, 1 \leq i \leq\lceil\operatorname{deg}(v) / 2\rceil$. Then use $\max _{i} s\left(X_{i}\right)+\left\lceil\Delta\left(G^{*}\right) / 2\right\rceil n$ searchers to clear each subgraph $X\left(T_{i}\right)$ separately. After that, we perform $S$ to clear $X(v)$. Each time after some $a_{i}$ is cleared by $S$, we remove the searcher on $a_{i}$ and place it on $a_{i}^{\prime}, 1 \leq i \leq\lceil\operatorname{deg}(v) / 2\rceil$. Finally, after $X(v)$ is cleared, we again use $\max _{i} s\left(X_{i}\right)+\left\lceil\Delta\left(G^{*}\right) / 2\right\rceil n$ searchers to clear each subgraph $X\left(T_{i}^{\prime}\right)$ separately. Therefore, we can clear $G$ with no more than $\max _{i} s\left(X_{i}\right)+\left\lceil\Delta\left(G^{*}\right) / 2\right\rceil n+\lceil\operatorname{deg}(v) / 2\rceil \leq \max _{i} s\left(X_{i}\right)+\left\lceil\Delta\left(G^{*}\right) / 2\right\rceil(n+1)$ searchers.

CAse 2. $G^{*}$ has an avenue $v_{1} v_{2} \ldots v_{t}, t>1$. Let $v_{0}$ be a neighbor of $v_{1}$ other than $v_{2}$ and let $v_{t+1}$ be a neighbor of $v_{t}$ other than $v_{t-1}$. Let $X\left(v_{i}\right), 0 \leq i \leq t+1$, be the subgraph of $G$ that corresponds to $v_{i}$. For $0 \leq i \leq t$, let $b_{i}$ be the vertex shared by $X\left(v_{i}\right)$ and $X\left(v_{i+1}\right)$. For $1 \leq i \leq t$, let $S_{i}$ be an optimal search strategy of $X\left(v_{i}\right)$ such that $b_{i-1}$ is cleared before $b_{i}$ is cleared. Thus, we can use a similar search strategy described in CASE 1 to clear each $X\left(v_{i}\right)$ and all the subgraphs that correspond to the edge-branches of $v_{i}$. Note that when we clear $X\left(v_{i}\right), b_{i-1}$ and $b_{i}$ form a pair as $a_{i}$ and $a_{i}^{\prime}$ in CASE 1 . In such a strategy we never need more than $\max _{i} s\left(X_{i}\right)+\left\lceil\Delta\left(G^{*}\right) / 2\right\rceil(n+1)$ searchers.

In Theorem 5.7, if each $X_{i}$ is a unicyclic graph, then we have a linear time approximation algorithm for cycle-disjoint graphs. We can design a linear time approximation algorithm when each $s\left(X_{i}\right)$ can be found in linear time.

\section{Conclusions}

Our work mainly involves four aspects. First, we establish a linear time algorithm to compute the search number and the optimal search strategy for a 3-cycle-disjoint graph using the labeling method. Second, 
we improve the running time of the algorithm for computing the vertex separation and the optimal layout of a unicyclic graph from $O(n \log n)$ to $O(n)$. For a graph $G$, the search number of $G$ equals the vertex separation of the 2-expansion of $G$. Thus, our improved algorithm can also compute the search number of a unicyclic graph. Third, we show how to compute the search number and the optimal search strategy of a $k$-ary cycle-disjoint graph. Finally, we prove several theorems that can be applied to design approximation algorithms for cycle-disjoint graphs and even more complicated graphs.

The results presented in the paper is a preliminary part of our research that will proceed to more complicated graphs with treewidth at most two. The roadmap we outlined was to find efficient algorithms to compute the search number of the following graphs one after another: trees, unicyclic graphs, CDGs and then outerplanar graphs. We have successfully found $O(n)$ algorithms for trees and unicyclic graphs and some classes of CDGs. In the future, finding efficient algorithms for computing the search number of graphs with constant treewidth continues to be a challenge.

\section{References}

[1] B. Alspach, Searching and searching graphs: A brief survey, Le Matematiche, 34pp.

[2] B. Alspach, X. Li, and B. Yang, Searching Graphs and Directed Graphs, Manuscript, 2004.

[3] H. Bodlaender, T. Kloks, Efficient and Constructive Algorithms for the Pathwidth and Treewidth of Graphs. J. Algorithms 21 (1996) 358-402.

[4] H. Bodlaender, F. Fomin, Approximation of pathwidth of outerplanar graphs. J. Algorithms 43 (2002) 190-200.

[5] D. Bienstock and P. Seymour, Monotonicity in graph searching, Journal of Algorithms 12 (1991) 239-245.

[6] J. Ellis, I. Sudborough and J. Turner, The vertex separation and search number of a graph, Information and Computation 113 (1994) 50-79.

[7] J. Ellis and M. Markov, Computing the vertex separation of unicyclic graphs, Information and Computation 192 (2004) 123-161.

[8] M. Garey and D. Johnson, Computers and Intractability: A Guide to the Theory of NP-Completeness, Freeman, San Francisco, 1979.

[9] N. Kinnersley, The vertex separation number of a graph equals its path-width, Information Processing Letters 42 (1992) 345-350.

[10] L. M. Kirousis and C. H. Papadimitriou, Searching and pebbling, Theoretical Computer Science 47 (1986) 205-218.

[11] A. S. LaPaugh, Recontamination does not help to search a graph. Journal of ACM 40 (1993) $224-245$.

[12] N. Megiddo, S. L. Hakimi, M. Garey, D. Johnson and C. H. Papadimitriou, The complexity of searching a graph, Journal of ACM 35 (1988) 18-44.

[13] T. Parsons, Pursuit-evasion in a graph. Theory and Applications of Graphs, Lecture Notes in Mathematics, Springer-Verlag, pp. 426-441, 1976.

[14] S. Peng, C. Ho, T. Hsu, M. Ko, and C. Tang, Edge and Node Searching Problems on Trees, Theoretical Computer Science 240 (2000) 429-446.

[15] B. Yang, D. Dyer, and B. Alspach, Searching graphs with large clique number, 35 pp (submitted). 